\section{Special Report 86-11}

June 1986
US Army Corps of Engineers

Cold Regions Research \& Engineering Laboratory

\title{
Concentration and flux of wind-blown snow
}

Malcolm Mellor and Gregor Fellers 


\begin{tabular}{|c|c|c|}
\hline \multicolumn{2}{|c|}{ REPORT DOCUMENTATION PAGE } & $\begin{array}{l}\text { READ INSTRUCTIONS } \\
\text { BEFORE COMPLETING FORM }\end{array}$ \\
\hline $\begin{array}{l}\text { 1. REPORT NUMBER } \\
\text { Special Report } 86-11\end{array}$ & 2. GOVT ACCESSION NO. & 3. RECIPIENT'S CATALOG NUMBER \\
\hline \multirow{2}{*}{\multicolumn{2}{|c|}{$\begin{array}{l}\text { 4. TITLE (end Subtitle) } \\
\text { CONCENTRATION AND FLUX OF } \\
\text { WIND-BLOWN SNOW }\end{array}$}} & 5. TYPE OF REPORT \& PERIOD COVERED \\
\hline & & 6. PERFORMING ORG. REPORT NUMBER \\
\hline \multicolumn{2}{|l|}{$\begin{array}{l}\text { 7. AUTHOR(s) } \\
\text { Malcolm Mellor and Gregor Fellers }\end{array}$} & 8. CONTRACT OR GRANT NUMBER(B) \\
\hline \multicolumn{2}{|l|}{$\begin{array}{l}\text { 9. PERFORMING ORGANIZATION NAME AND ADDRESS } \\
\text { U.S. Army Cold Regions Research and } \\
\text { Engineering Laboratory } \\
\text { Hanover, New Hampshire } 03755-1290\end{array}$} & $\begin{array}{l}\text { 10. PROGRAM ELEMENT, PROJECT, TASK } \\
\text { AREA \& WORK UNIT NUMBERS. } \\
6.27 .30 \text { A } \\
4 \text { A } 762730 \text { AT } 42 \\
\text { BS } 044\end{array}$ \\
\hline \multirow{2}{*}{\multicolumn{2}{|c|}{$\begin{array}{l}\text { 11. CONTROLLING OFFICE NAME AND ADDRESS } \\
\text { Office of the Chief of Engineers } \\
\text { Washington, D.C. } 20314-1000\end{array}$}} & $\begin{array}{l}\text { 12. REPORT DATE } \\
\text { Jüne } 1986 \\
\end{array}$ \\
\hline & & $\begin{array}{l}\text { 13. NUMBER OF PAGES } \\
20\end{array}$ \\
\hline \multirow{2}{*}{\multicolumn{2}{|c|}{ 14. MONITORING AGENCY NAME \& ADDRESS(If different from Controlling Office) }} & $\begin{array}{l}\text { 15. SECURITY CLASS. (of this report) } \\
\text { Unclassified }\end{array}$ \\
\hline & & $\begin{array}{l}\text { 15a. DECLASSIFICATION/DOWNGRADING } \\
\text { SCHEDULE }\end{array}$ \\
\hline
\end{tabular}

Approved for public release; distribution is unlimited.

17. DISTRIBUTION STATEMENT (of the abstract ontered in Block 20, if different from Report)

18. SUPPLEMENTARY NOTES

19. KEY WORDS (Continue on reverse side if necossary and identify by block number)

Obscuration

Visibility

Snow Wind tunnel models

Snowdrifts

Snow removal

20. ABSTRACT (Continue an roveras stids if mecosssary and tdontify by block number)

Representative graphical relations are developed for the flux and concentration of windblown snow as functions of wind speed and height above surface. Previously published field data are tabulated to provide 1201 data sets for flux and the same number for mass concentration. Using appropriately transformed variables, multiple regression analysis yields empirical relations for horizontal mass flux as a function of wind speed and height, and for mass concentration as a function of wind speed and height. 
PREFACE

This report was prepared by Dr. Malcolm Mellor, Research Physical Scientist, Experimental Engineering Division, and Gregor Fellers, Computer Specialist, Engineering and Measurement Services Branch, Technical Services Division, U.S. Army Cold Regions Research and Engineering Laboratory. The work was supported as part of DA Project 4A762730AT42, Design, Construction, and Operations Technology for Cold Regions, Task Area BS (Base Support), Work Unit 044, Contro1 of Snowdrifting and Ice Accretion.

Technical review of the manuscript was performed by $\mathrm{Dr}$. Edgar Andreas of CRREL and Dr. Uwe Radok. 


\section{CONCENTRATION AND FLUX OF WIND-BLOWN SNOW \\ Ma1colm Me1lor and Gregor Fellers}

Information on the mass concentration and the horizontal mass flux of blowing snow is useful in dealing with certain practical problems, notably those involving snowdrift formation, wind tunnel (or water flume) modeling of blowing snow, reduction of visibility, and attenuation of radiation near optical frequencies. The basic requirement is for data on the concentration and flux as functions of height and wind speed when the wind blows across a flat surface with an ample supply of loose snow particles.

Many years ago, Australian investigators working in Antarctica gathered a large quantity of systematic data on blowing snow, using techniques and equipment that were validated by tests and theory. Results were analyzed very thoroughly, with emphasis on establishment of the relevant physics. Without doing anything essentially different from the original investigators, we have combined the Australian data and applied multiple regression analysis in order to relate mass flux and mass concentration to the relevant variables that are most easily measured, i.e. height above surface, and wind speed at standard anemometer height.

The Australian studies were inspired by Fritz Loewe, whose interest in blizzards was. stimulated by a wintering in Terre Adelie. Systematic observations were originated by $M$. Mellor and developed under the direction of U. Radok. The great majority of the field observation were made by $R$. Dingle, and analyzed by $U$. Radok and W. Budd. Anemometers and aerodynamic snow collectors were mounted in pairs on vertical masts, with logarithmic vertical spacing. At each level, the instruments gave wind speed and mass flux. Mass concentration and the parameters of the wind profile (shear velocity and roughness height) could then be derived. Altogether, 1201 usable data sets were obtained (see Appendix). They include measurements in conditions where: (a) there is new snow falling directly into the turbulent boundary layer, and (b) there is no precipitation from above and all particles are picked up from the surface. Most of the observational 
data have been published (Budd et al. 1965, Me1lor and Radok 1960), but some unpublished results were provided directly by Dingle and Radok. Information on particle size and fall velocity can be found elsewhere (Budd 1965, Budd et al. 1965, Mellor 1965); with strong winds (>10 m/s) mean particle size is approximately $100 \mu \mathrm{m}$ around $1 \mathrm{~m}$ height, about 150-200 $\mu \mathrm{m}$ within a few centimetres of the surface, and about $90 \mu \mathrm{m}$ at head height.

Measurements have been made by other investigators, but we do not have access to the actual observational data, or to the instrument calibrations that are needed to establish comparability.

To obtain representative empirical relations for the dependence of flux $q$ and concentration $\rho$ on height $z$ and wind speed $u_{10}$, we can perform multiple regression analysis on the data while taking account of the relevant physics. The dependent variable $Y$ is a simple function of either $q$ or $\rho$, and it is expressed initially as a 10-term polynomial with cross-products and terms up to the third power. Some terms may be discarded on the basis of significance tests. The two independent variables $x_{1}$ and $x_{2}$ are simple functions of $z$ and $u_{10}$ respectively. If $Y$ is taken directly as $\rho$, with $x_{1}$ and $x_{2}$ as $z$ and $u_{10}$ respectively, the correlation is very poor, since the observed values of $\mathrm{Y}$ range over more than 4 orders of magnitude, while corresponding values of $x_{1}$ range over more than 2 orders of magnitude. Using the logarithms of the observed quantities for $Y$ and $X_{1}$, together with $u_{10}$ as $x_{2}$, the results are much better. However, both theory and observation (Dingle and Radok 1961, see also Budd et al. 1965 and Mellor 1965) indicate that the relation between $1 n \rho$ and the reciprocal of wind speed, $1 / \mathrm{u}_{10}$, should be close to linear, and it is found that regression of $\ln \rho(Y)$ against $\ln z\left(X_{1}\right)$ and $1 / u_{10}\left(X_{2}\right)$ gives the best fit to the data.

A parallel treatment for the regression of $1 \mathrm{n} q$ against $1 \mathrm{n} z$ and $1 / \mathrm{u}_{10}$ is less easy to relate to theory. However, by using different forms of the wind profile in the expressions for $\rho$ and $u$ in the product $q=\rho u$, the logarithmic regression forms for $q$ and $z$ can be justified (see Mellor 1965, p. 15, eq 17). By comparing magnitudes for the two parts of the exponent in the hybrid equation for $q$, it can be argued that the wind-dependence of $q$ is not radically different from that of $\rho$, so that there can be no strong objection to taking $x_{2}$ as $1 / u_{10}$ in the regression. 
In both regressions the multiple correlation coefficient $r=0.978$. The standard error of $\mathrm{Y}$ about the regression plane is 0.453 for in $\rho$ and 0.447 for $\ln q$ (with $\rho$ and $q$ in units of $\mathrm{g} / \mathrm{m}^{3}$ and $\mathrm{g} / \mathrm{m}^{2}-\mathrm{s}$ respectively).

The final regression equations were as follows.

Mass flux, q

$$
\begin{aligned}
\mathrm{Y} & =\ln \mathrm{q}, \mathrm{X}_{1}=\ln \mathrm{z}, \mathrm{X}_{2}=1 / \mathrm{u}_{10} . \quad\left(\mathrm{q} \text { in } \mathrm{g} / \mathrm{m}^{2}-\mathrm{s}, \mathrm{z} \text { in } \mathrm{m}, \mathrm{u}_{10} \text { in } \mathrm{m} / \mathrm{s}\right) \\
\mathrm{Y} & =10.089-0.41049 \mathrm{x}_{1}-122.03 \mathrm{x}_{2}-0.13856 \mathrm{x}_{1}{ }^{2}-14.446 \mathrm{x}_{1} \mathrm{X}_{2} \\
& -0.0059773 \mathrm{x}_{1}^{3}+3.2682 \mathrm{x}_{1}{ }^{2} \mathrm{X}_{2}+114.13 \mathrm{x}_{1} \mathrm{X}_{2}{ }^{2}+2290.0 \mathrm{x}_{2}{ }^{3}
\end{aligned}
$$

Mass concentration, $\rho$

$$
\begin{aligned}
Y & =\ln \rho, X_{1}=1 n z, X_{2}=1 / u_{10} \cdot \quad\left(\rho \text { in } g / m^{3}, z \text { in } m, u_{10} \text { in } m / s\right) \\
Y & =4.8679-0.42209 X_{1}-34.369 X_{2}-0.13265 X_{1}{ }^{2}-17.427 X_{1} X_{2} \\
& -972.01 X_{2}{ }^{2}-0.0070277 X_{1}^{3}+3.2692 X_{1}{ }^{2} X_{2}+135.54 X_{1} X_{2}{ }^{2} \\
& +6430.2 X_{2}{ }^{3}
\end{aligned}
$$

Figure 1 shows probable values of mass concentration $\rho$ as a function of height $z$ and wind speed $u_{10}$ according to the regression equation. In Figure 1a, $\rho$ is plotted against $z$ on logarithmic scales, with $u_{10}$ as parameter. In Figure $1 b, \rho$ is plotted on a logarithmic scale against $1 / \mathrm{u}_{10}$ (or against $u_{10}$ on a distorted scale), with $z$ as parameter. The observational range for $z$ was from 0.03 to $4 \mathrm{~m}$, and for $u_{10}$ it was mainly within the range 10 to $25 \mathrm{~m} / \mathrm{s}$, with a $\mathrm{few}$ values up to $36 \mathrm{~m} / \mathrm{s}$.

For high wind speeds, when particles are well diffused, Figure la indicates that the theoretical power relation between $\rho$ and $z$ is a good approximation, with an exponent not far from -1 . The exponent given by simple theory is $-w / k_{*}$, where $w$ is particle fall velocity, $u_{*}$ is shear velocity ( $\approx 1 \mathrm{~m} / \mathrm{s}$ with winds of $25-35 \mathrm{~m} / \mathrm{s}$ ), and $k$ is von Karman's constant $(0.4)$. If $-w / k u_{*} \approx-1$ for strong winds, $w \approx 0.4 \mathrm{~m} / \mathrm{s}$, which is a credible value for particles of wind-blown snow.

At low wind speeds, when there is a sorting of particle size (and fall velocity) with height (see Budd 1965), a linear relation between in $\rho$ and In $z$ applies on ly at low levels. At the lowest observed wind speeds $(\approx 10$ $\mathrm{m} / \mathrm{s}), \rho$ tends to a limit of about $0.06 \mathrm{~g} / \mathrm{m}^{3}$ when $z$ is greater than a few metres. This is the sort of concentration that corresponds to very light snowfall in calm weather. At the lower levels, the general (negative) 


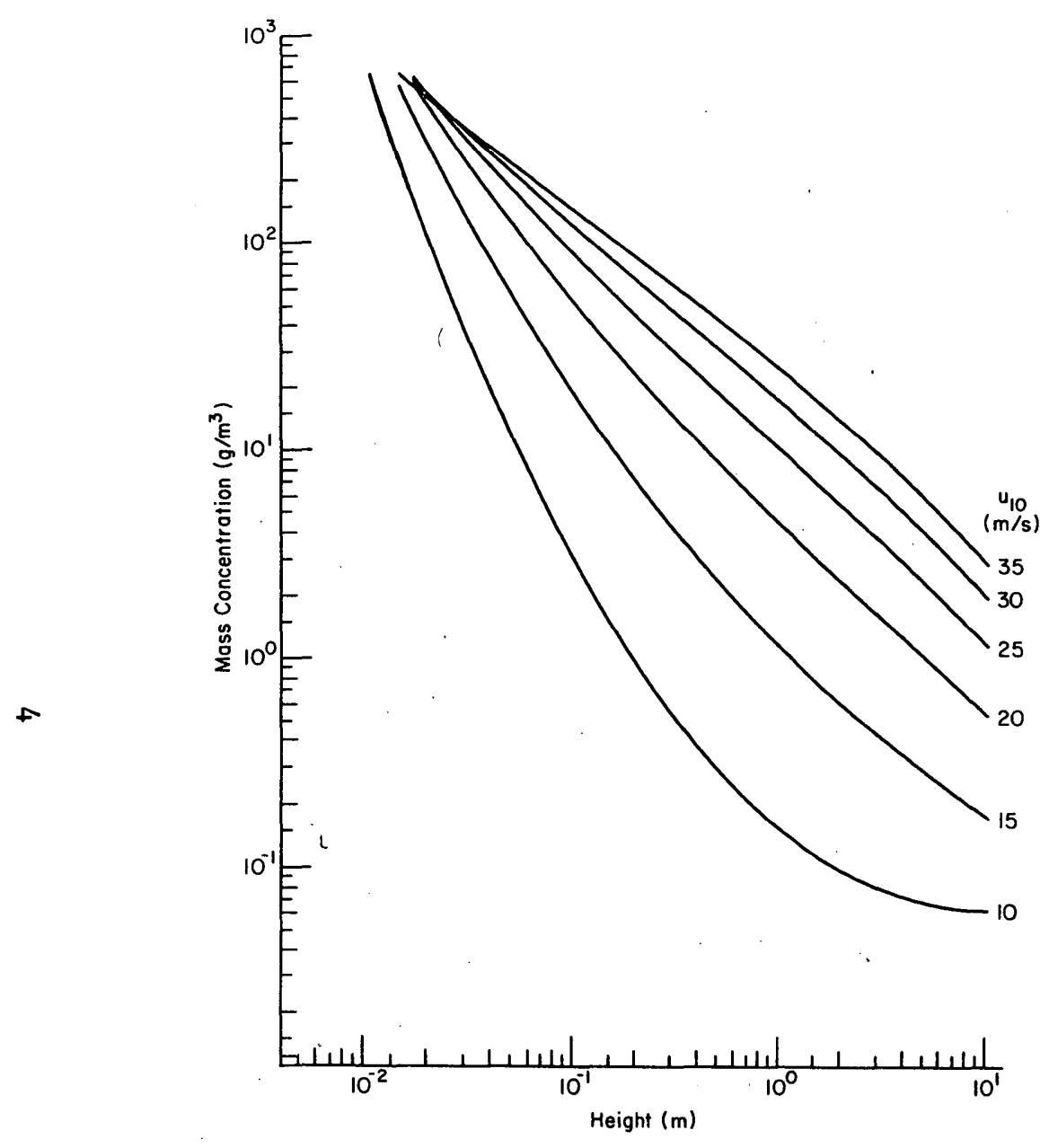

a. $\rho$ plotted against $z$ on logarithmic scales, with $\mathrm{u}_{10}$ as parameter.

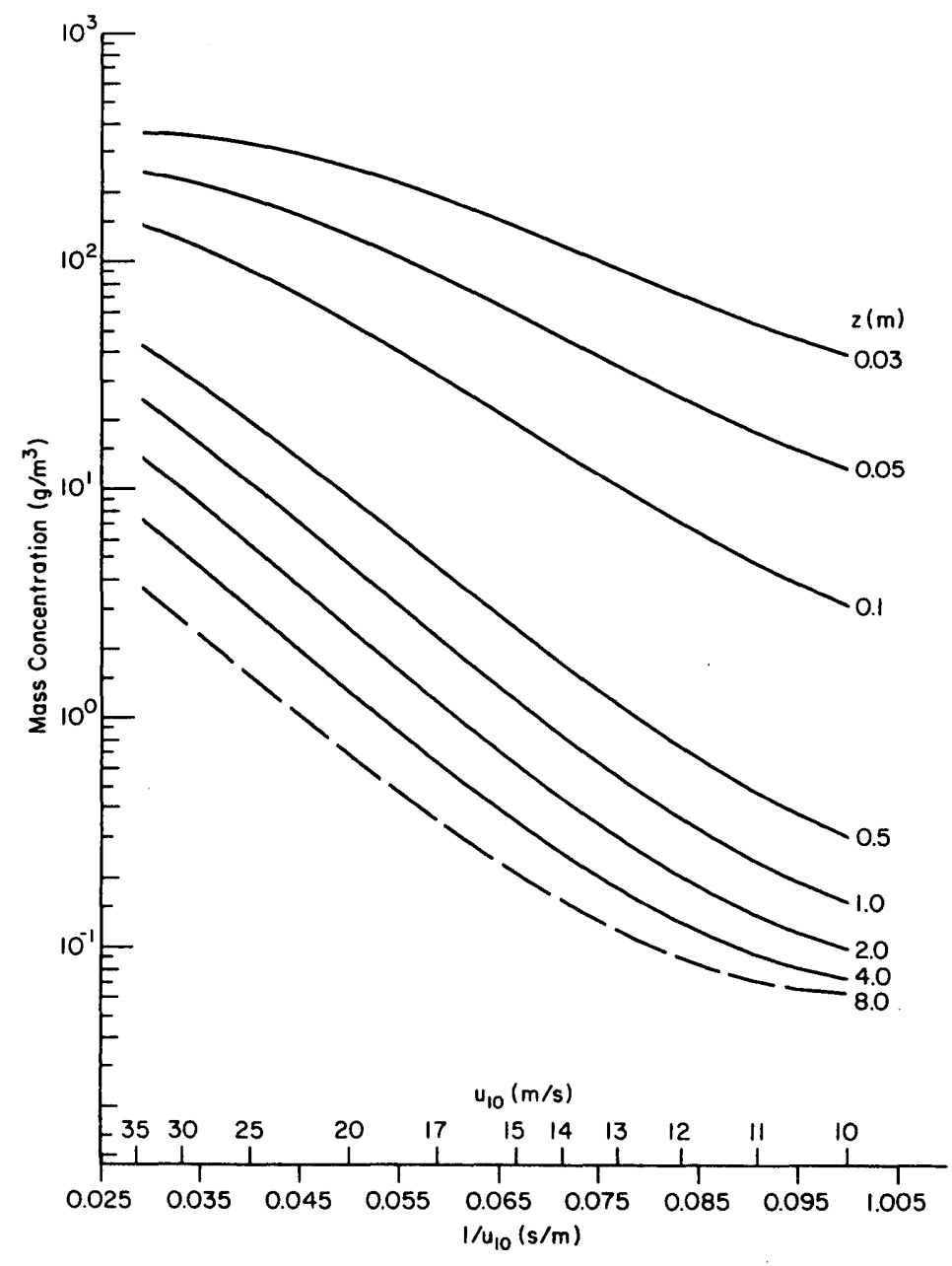

b. o (logarithmic scale) plotted against $1 / u_{10}$ (linear scale) and $u_{10}$ (distorted scale), with $z$ as parameter. The line for $z=8 \mathrm{~m}$ is broken because the highest measuring level was $z=4 \mathrm{~m}$.

Figure 1. Probable values of mass concentration $\rho$ as a function of height $z$ and wind speed $u_{10} \cdot$ 
slope of the curves in Figure la increases as $u_{10}$ decreases, presumably because the shear velocity is proportional to $u_{10}$. Very close to the ground $(z \approx 10 \mathrm{~mm})$, the curves converge to a focus, indicating an upper limit of concentration that is not strongly dependent on wind speed. This limit, which is approximate $1 \mathrm{y} 1400 \mathrm{~g} / \mathrm{m}^{3}$ and therefore close to air density $\left(\approx 1300 \mathrm{~g} / \mathrm{m}^{3}\right)$, was predicted and noted in earlier studies (Owen 1964, Budd et al. 1965, Greeley and Iversen 1985). The height of the "focus" is more or less the height of the top of the saltation layer.

Figure $1 \mathrm{~b}$ shows that the theoretical expectation of inverse proportionality between $1 \mathrm{n} \rho$ and $1 / \mathrm{u}_{10}$ is realized in strong winds at levels above $0.5 \mathrm{~m}$. However, with less strong winds the concentration does not decrease as much as might be expected from simple theory, perhaps because falling snow sets a lower limit for $\rho$. At low levels, $\rho$ becomes progressively less sensitive to wind speed, as already mentioned in connection with the "focus" in Figure 1a. The curves for low levels in Figure 1b could perhaps be approximated by straight lines, but they actually show contraflexure. Taken at face value, they suggest that $\rho$ may become almost independent of $\mathrm{u}_{10}$ for very high winds and for layers very close to the surface, a trend which seems credible. They also indicate a trend towards a lower limit of $\rho$ in light winds for layers very close to the surface. This is more difficult to rationalize, although one might consider systematic change in particle size as a possible explanation.

Figure 2 gives horizontal mass flux $q$ as a function of height $z$ and wind speed $u_{10}$. The plot of $\ln q$ against $\ln z$ (Fig. 2a) shows approximate linearity for high wind speeds, with the relation not too far from inverse proportionality. In lighter winds, an approximately linear relation between $\ln q$ and $1 \mathrm{n} z$ prevails only at low levels. At high levels and low wind speeds, $q$ for a given value of $z$ tends towards a limit. As was the case for $\rho$, the curves in Figure 2a tend to suggest convergence to a focus very close to the surface, but it is not easy to accept this trend. Extension of the curves to a common point indicates $q \approx 30,000 \mathrm{~g} / \mathrm{m}^{2}-\mathrm{s}$ at $z \approx 6$ $\mathrm{mm}$. However, the limiting value of $\rho$ at low level should be approximately $1300 \mathrm{~g} / \mathrm{m}^{3}$, with a wind speed of approximate $1 \mathrm{y} 2 \mathrm{~m} / \mathrm{s}$ very near the surface. This is an order of magnitude discrepancy; the expected maximum for very low levels is $\mathrm{q} \approx 3,000 \mathrm{~g} / \mathrm{m}^{2}-\mathrm{s}$ at $\mathrm{z}<10 \mathrm{~mm}$. 


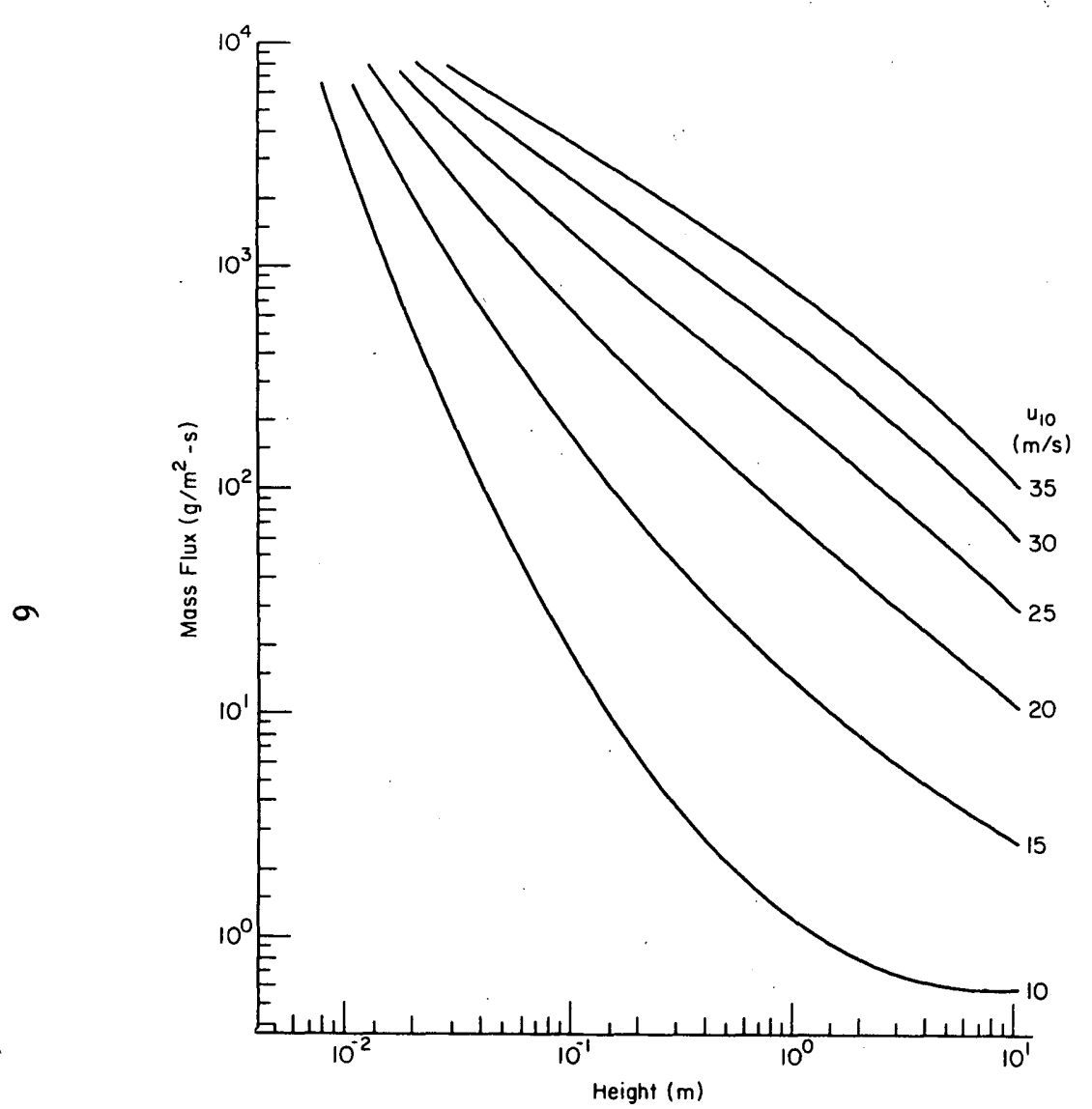

a. $q$ plotted against $z$ on logarithmic scales, with $u_{10}$ as parameter.

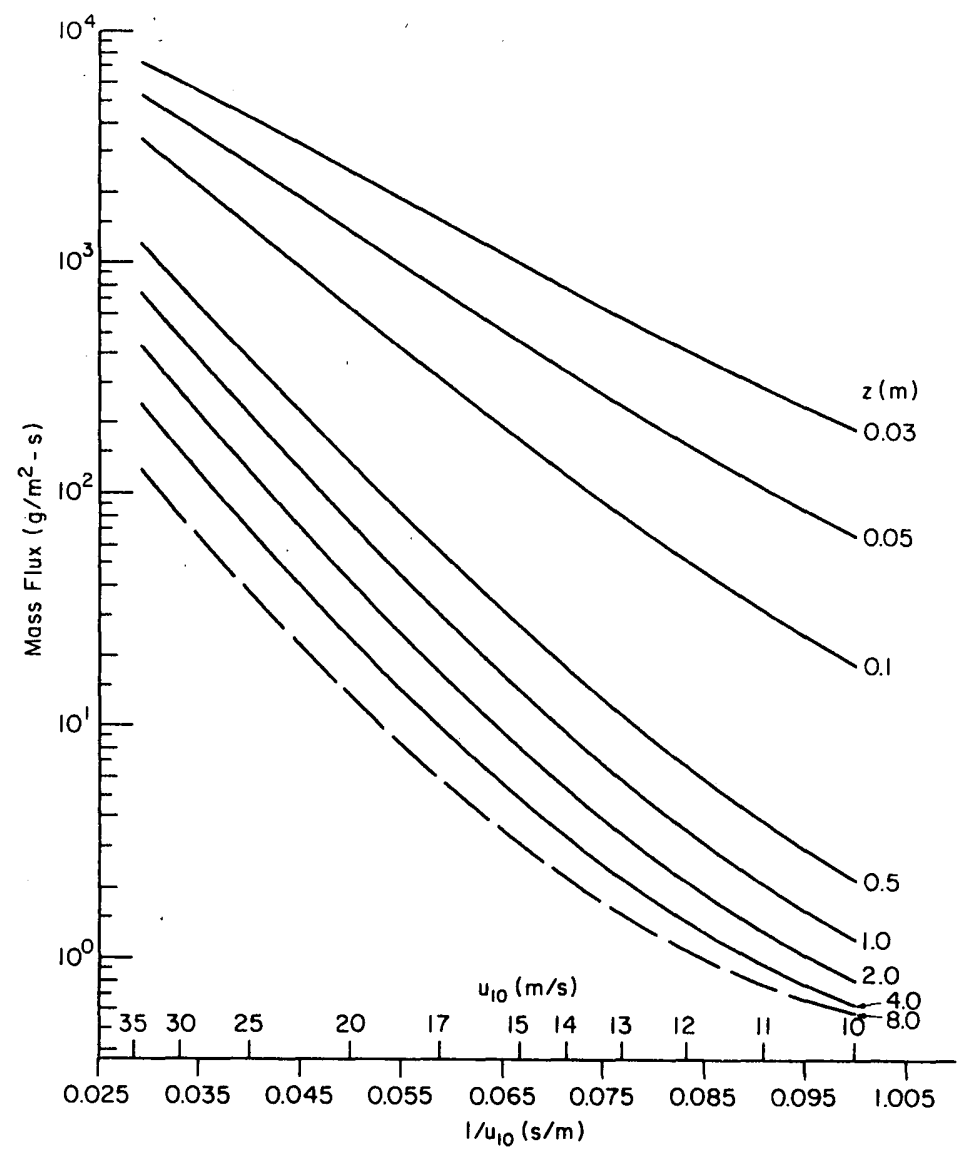

b. q (logarithmic scale) plotted against $1 / \mathrm{u}_{10}$ (linear scale) and $u_{10}$ (distorted scale), with $z$ as parameter. The line for $z=8 \mathrm{~m}$ is broken because the highest measuring level was $z=4 \mathrm{~m}$.

Figure 2. Probable values of horizontal mass flux $q$ as a function of height $z$ and wind speed $u_{10}$. 
Figure $2 \mathrm{~b}$ shows approximate inverse proportionality between $\ln \mathrm{q}$ and $1 / \mathrm{u}_{10}$ at very low levels, but above $0.1 \mathrm{~m}$ there is inverse proportionality only in very strong winds. At the higher levels, there appears to be a trend towards a lower limit of $q$ as $u_{10}$ decreases. The indication is that such a limit might be of order 0.1 to $1.0 \mathrm{~g} / \mathrm{m}^{2}-\mathrm{s}$, corresponding to the suggested lower $11 \mathrm{mit}$ of $\rho$ around $0.06 \mathrm{~g} / \mathrm{m}^{3}$ if wind speeds are a $\mathrm{f}$ ew metres per second.

If new measurements should be made in the future, it would be useful to extend the profiles above the 4-m level, since extrapolation to high levels is called for in the treatment of certain problems.

\section{References}

Budd, W. (1965) The drifting of non-uniform snow particles. In Antarctic Research Series, vol. 7 (M. Rubin, Ed.), American Geophysical Union.

Budd, W., R. Dingle and U. Radok (1965) The Byrd snow drift project: Outline of basic results. In Antarctic Research Series, vo1. 7 (M. Rubin, Ed.), American Geophysical Union.

Dingle, W.R.J. and U. Radok (1961) Antarctic snow drift and mass transport. General Assembly of Helsinki, International Association of Scientific Hydrology, International Union of Geodesy and Geophysics, pub. no. 55 .

Greeley, R. and J.D. Iversen (1985) Wind as a geological process on Earth, Mars, Venus and Titan. Cambridge University Press.

Mellor, M. and U. Radok (1960) Some properties of drifting snow. In Antarctic Meteorology, Oxford: Pergamon Press.

Mellor, M. (1965) Blowing snow. U.S. Army Cold Regions Research and Engineering Laboratory, Monograph III-A3c.

Owen, P.R. (1964) Saltation of uniform sand grains in air. Journal of Fluid Mechanics, 20(2): 225-242. 
Principal data source: Budd et al. (1965). Additional unpublished data provided by R. Dingle and U. Radok. Some data for very strong winds from Mellor and Radok (1960).

\begin{tabular}{|c|c|c|c|c|c|c|c|c|c|c|}
\hline $\begin{array}{l}\text { He i ght } \\
\text { (z) }\end{array}$ & $\begin{array}{l}\text { wind } \\
\text { e } 10 \mathrm{~m}\end{array}$ & $\begin{array}{l}\text { Mass } \\
\text { Conc }\end{array}$ & $\begin{array}{l}\text { Wind } \\
\text { e z m }\end{array}$ & $\begin{array}{l}\text { Mass } \\
\text { Flux }\end{array}$ & & $\begin{array}{l}\text { Height } \\
(z)\end{array}$ & $\begin{array}{l}\text { Wind } \\
\text { e } 10 \mathrm{~m}\end{array}$ & $\begin{array}{l}\text { Mass } \\
\text { Conc }\end{array}$ & $\begin{array}{l}\text { Wind } \\
Q=\mathrm{m}\end{array}$ & $\begin{array}{l}\text { Mass } \\
\text { Flux }\end{array}$ \\
\hline 4.000 & 9.60 & 0.076 & 8.79 & 0.668 & & 2.000 & $\cdot 12.66$ & 0.564 & 11.00 & 6.204 \\
\hline 4.000 & 9.78 & 0.019 & 8.75 & 0.170 & & 2.000 & 12.68 & 0.255 & 11.04 & 2.815 \\
\hline 4.000 & 10.19 & 0.111 & 9.37 & 1.040 & & 1.000 & 9.60 & 0.175 & 7.59 & 1.328 \\
\hline 4.000 & 10.73 & 0.057 & 9.93 & 0.566 & & 1.000 & 9.78 & 0.037 & 7.54 & 0.279 \\
\hline 4.000 & 11.07 & 0.136 & 10.28 & 1.398 & & 1.000 & 10.19 & 0.251 & 8.23 & 2.066 \\
\hline 4.000 & 11.17 & 0.219 & 10.16 & 2.225 & & 1.000 & 10.73 & 0.130 & 8.59 & 1.117 \\
\hline 4.000 & 11.21 & 0.082 & 10.23 & 0.839 & & 1.000 & 11.07 & 0.264 & 9.59 & 2.532 \\
\hline 4.000 & 11.31 & 0.109 & 10.33 & 1.126 & & 1.000 & 11.17 & 0.505 & 8.79 & 4.439 \\
\hline 4.000 & 11.60 & 0.104 & 10.78 & 1.121 & & 1.000 & 11.21 & 0.380 & 8.90 & 3.382 \\
\hline 4.000 & 11.64 & 0.105 & 10.62 & 1.115 & & 1.000 & 11.31 & 0.385 & 8.60 & 3.311 \\
\hline 4.000 & 11.64 & 0.210 & 10.75 & 2.257 & & 1.000 & 11.60 & 0.199 & 9.29 & 1.849 \\
\hline 4.000 & 11.82 & 0.046 & 10.85 & 0.499 & & 1.000 & 11.64 & $0.424^{\circ}$ & 9.24 & 3.918 \\
\hline 4.000 & 11.87 & 0.120 & 10.83 & 1.300 & & $1.000^{\circ}$ & 11.64 & 0.691 & 9.36 & 6.468 \\
\hline 4.000 & 11.93 & 0.207 & 10.85 & 2.246 & & 1.000 & 11.82 & 0.278 & 9.44 & 2.624 \\
\hline 4.000 & 11.94 & 0.103 & 10.77 & 1.109 & & 1.000 & 11.87 & 0.436 & 9.56 & 4.168 \\
\hline 4.000 & 12.08 & 0.103 & 10.73 & 1.105 & & 1.000 & 11.93 & 0.362 & 9.26 & 3.352 \\
\hline 4.000 & 12.10 & 0.025 & 11.18 & 0.279 & & 1.000 & 11.94 & 0.423 & 9.30 & $3.934^{\circ}$ \\
\hline 4.000 & 12.19 & 0.150 & 11.22 & 1.683 & & 1.000 & 11.08 & 0.218 & 9.21 & 2.008 \\
\hline 4.000 & 12.26 & 0.250 & 11.27 & 2.818 & & 1.000 & 12.10 & 0.205 & 9.47 & 1.941 \\
\hline 4.000 & 12.29 & 0.233 & 11.28 & 2.628 & & 1.000 & 12.19 & 0.522 & 9.71 & 5.069 \\
\hline 4.000 & 12.41 & 0.050 & 10.99 & 0.549 & & 1.000 & 12.26 & 0.406 & 9.69 & 3.934 \\
\hline 4.000 & 12.41 & 0.249 & 11.25 & 2.801 & & 1.000 & 12.29 & 0.348 & 9.67 & 3.365 \\
\hline 4.000 & .12 .43 & 0.140 & 11.42 & 1.599 & & 1.000 & 12.41 & 0.366 & 9.34 & 3.418 \\
\hline 4.000 & 12.52 & 0.045 & 11.65 & 0.524 & & 1.000 & 12.41 & 0.759 & 9.62 & 7.302 \\
\hline 4.000 & 12.56 & 0.582 & 11.53 & 6.710 & & 1.000 & 12.43 & 0.484 & 9.73 & 4.709 \\
\hline 4.000 & 12.56 & 0.161 & 11.70 & 1.884 & & 1.000 & 12.52 & 0.163 & 10.29 & 1.677 \\
\hline 4.000 & 12.66 & 0.264 & 11.74 & 3.099 & & 1.000 & 12.56 & 0.821 & 9.74 & 7.997 \\
\hline 4.000 & 12.68 & 0.095 & 11.84 & 1.125 & & 1.000 & 12.56 & 0.553 & 10.14 & 5.607 \\
\hline 2.000 & 9.60 & 0.122 & 8.43 & 1.028 & & 1.000 & 12.66 & 0.906 & 10.07 & 9.123 \\
\hline 2.000 & 9.78 & 0.031 & 8.28 & 0.257 & & 1.000 & 12.68 & 0.544 & 10.05 & 5.467 \\
\hline 2.000 & 10.19 & 0.176 & 8.90 & 1.566 & & 0.500 & 9.60 & 0.331 & 7.11 & 2.353 \\
\hline 2.000 & 10.73 & 0.091 & 9.17 & 0.834 & & 0.500 & 9.78 & 0.120 & 6.99 & 0.839 \\
\hline 2.000 & 11.07 & 0.230 & 9.79 & 2.252 & & 0.500 & 10.19 & 0.271 & 7.61 & 2.062 \\
\hline 2.000 & 11.17 & 0.410 & 9.75 & 3.997 & & 0.500 & 10.73 & 0.354 & 7.92 & 2.804 \\
\hline 2.000 & 11.21 & 0.205 & 9.61 & 1.970 & . & 0.500 & 11.07 & 0.541 & 8.82 & 4.772 \\
\hline 2.000 & 11.31 & 0.236 & 9.57 & 2.259 & & 0.500 & 11.17 & 0.889 & 8.32 & 7.396 \\
\hline 2.000 & 11.60 & 0.149 & 10.08 & 1.502 & & 0.500 & 11.21 & 0.791 & 8.06 & 6.375 \\
\hline 2.000 & 11.64 & 0.226 & 10.11 & 2.285 & & 0.500 & 11.31 & 0.422 & 7.48 & 3.157 \\
\hline 2.000 & 11.64 & 0.336 & 10.02 & 3.367 & & 0.500 & 11.60 & 0.363 & 8.77 & 3.184 \\
\hline 2.000 & 11.82 & 0.074 & 10.20 & 0.755 & & 0.500 & 11.64 & 0.855 & 8.46 & 7.233 \\
\hline 2.000 & 11.87 & 0.221 & 10.19 & 2.252 & - & 0.500 & 11.64 & 1.133 & 8.57 & 9.710 \\
\hline 2.000 & 11.93 & 0.223 & 10.20 & 2.275 & & 0.500 & 11.82 & 0.420 & 8.69 & 3.650 \\
\hline 2.000 & 11.94 & 0.223 & 10.25 & 2.286 & & 0.500 & 11.87 & 0.861 & 8.65 & 7.448 \\
\hline 2.000 & 12.08 & 0.141 & $9: 94$ & 1.402 & & 0.500 & 11.93 & 0.790 & 8.54 & 6.747 \\
\hline 2.000 & 12.10 & 0.068 & 10.32 & 0.702 & & 0.500 & 11.94 & 0.659 & 8.44 & 5.562 \\
\hline 2.000 & 12.19 & 0.296 & 10.37 & 3.070 & & 0.500 & 12.08 & 0.590 & 8.09 & 4.773 \\
\hline 2.000 & 12.26 & 0.322 & 10.40 & 3.349 & & 0.500 & 12.10 & 0.478 & 8.65 & 4.135 \\
\hline 2.000 & 12.29 & 0.286 & 10.41 & 2.977 & & 0.500 & 12.19 & 1.006 & 8.88 & 8.933 \\
\hline 2.000 & 12.41 & 0.110 & 10.23 & 1.125 & & 0.500 & 12.26 & 0.566 & 8.99 & 5.088 \\
\hline 2.000 & 12.41 & 0.429 & 10.53 & 4.517 & & 0.500 & 12.29 & 0.464 & 8.85 & 4.106 \\
\hline 2.000 & 12.43 & 0.241 & 10.86 & 2.617 & & 0.500 & 12.41 & 0.544 & 8.26 & 4.493 \\
\hline 2.000 & 12.52 & 0.068 & 10.87 & 0.739 & & 0.500 & 12.41 & 1.151 & 8.77 & 10.094 \\
\hline 2.000 & 12.56 & 0.759 & 10.93 & 8.296 & & 0.500 & 12.43 & 0.734 & 9.11 & 6.687 \\
\hline 2.000 & 12.56 & 0.275 & 10.75 & 2.956 & & 0.500 & 12.52 & 0.507 & 9.76 & 4.948 \\
\hline
\end{tabular}




\begin{tabular}{|c|c|c|c|c|c|c|c|c|c|c|c|c|c|c|c|}
\hline & $\begin{array}{l}\text { Height } \\
\text { (z) }\end{array}$ & $\begin{array}{l}\text { Wind } \\
\text { e } 10 \mathrm{~m}\end{array}$ & $\begin{array}{l}\text { Mass } \\
\text { Conc }\end{array}$ & $\begin{array}{l}\text { Wind } \\
\text { e } z \mathrm{~m}\end{array}$ & $\begin{array}{l}\text { Mass } \\
\text { Flux }\end{array}$ & $\begin{array}{l}\text { the ight } \\
\text { (z) }\end{array}$ & $\begin{array}{l}\text { Wind } \\
\text { e } 10 \mathrm{~m}\end{array}$ & $\begin{array}{l}\text { Mass } \\
\text { Conc }\end{array}$ & $\begin{array}{l}\text { Wind } \\
\text { e } 2 \mathrm{~m}\end{array}$ & $\begin{array}{l}\text { Misss } \\
\text { Flux }\end{array}$ & $\begin{array}{l}\text { theight } \\
(i 2)\end{array}$ & $\begin{array}{l}\text { Wind } \\
10 \mathrm{~m}\end{array}$ & $\begin{array}{l}\text { Mas; } \\
\text { Cons: }\end{array}$ & $\begin{array}{l}\text { Wind } \\
\text { e } 2 \mathrm{~m}\end{array}$ & $\begin{array}{l}\text { Mass } \\
\text { Fi!11 }\end{array}$ \\
\hline & 0.500 & 12.56 & 1.359 & 9.30 & 12.639 & 0.125 & 12.43 & $\therefore .182$ & 7.68 & 47.478 & 0.031 & 12.41 & 176.590 & 4.41 & 779.203 \\
\hline & 0.500 & 12.56 & 1.034 & 9.44 & 9.761 & 0.125 & 12.52 & 4.849 & 8.26 & 40.053 & 0.031 & 12.41 & 57.070 & 5.4 .3 & 309.890 \\
\hline & 0.500 & 12.66 & 1.629 & 9.44 & 15.378 & 0.125 & 12.56 & 7.305 & 7.89 & 57.636 & 0.031 & 12.43 & 72.670 & 6.18 & 449.101 \\
\hline & 0.500 & 12.68 & 0.877 & 9.66 & 8.472 & 0.125 & 12.56 & 6.543 & 7.95 & 52.017 & 0.031. & 12.52 & 89.350 & 5.91 & 617.478 \\
\hline & 0.250 & 9.60 & 1.232 & 6.60 & 8.131 & 0.125 & 12.66 & 7.288 & $8.0:$ & 58.377 & 0.031 & 12.56 & 51.030 & 6.41 & 327.102 \\
\hline & 0.250 & 9.78 & 0.221 & 6.43 & 1.421 & 0.125 & 12.68 & 5.909 & 8.17 & 48.277 & 0.031 & 12.56 & 56.170 & 6.49 & 364.543 \\
\hline & 0.250 & 10.19 & 0.660 & 7.08 & 4.673 & 0.062 & 9.60 & 8.084 & 5.42 & 43.815 & 0.031 & 12.66 & 70.860 & 6.54 & 463.424 \\
\hline & 0.250 & 10.73 & 0.730 & 7.34 & 5.358 & 0.062 & 9.78 & 6.605 & 5.05 & 33.355 & 0.031 & 12.68 & 102.200 & 6.74 & 688.828 \\
\hline & 0.250 & 11.07 & 1.172 & 8.14 & 9.540 & 0.062 & 10.19 & 7.201 & 5.89 & 42.414 & 4.000 & .12 .70 & 0.096 & 11.77 & 1.130 \\
\hline & 0.250 & 11.17 & 1.568 & 7.45 & 11.682 & 0.062 & 10.73 & 10.088 & 6.00 & 60.528 & 4.000 & 12.75 & 0.128 & 11.76 & 1.505 \\
\hline & 0.250 & 11.21 & 1.691 & 7.63 & 12.902 & 0.062 & 11.07 & 11.726 & 7.16 & 83.958 & 4.000 & 12.77 & 0.143 & 11.84 & 1.693 \\
\hline & 0.250 & 11.31 & 1.012 & 7.27 & 7.357 & 0.062 & 11.17 & 11.899 & 6.15 & 73.179 & 4.000 & 12.84 & 0.348 & 11.87 & 4.131 \\
\hline & 0.250 & 11.60 & 0.671 & 7.97 & 5.348 & 0.062 & 11.21 & 13.290 & 5.99 & 79.607 & 4.000 & 12.85 & 0.158 & 11.85 & 1.872 \\
\hline & 0.250 & 11.64 & .1 .794 & 7.86 & 14.101 & 0.062 & 11.31 & 30.298 & 5.67 & 171.790 & 4.000 & 12.90 & 0.118 & 12.00 & 1.416 \\
\hline & 0.250 & 11.64 & 2.180 & 8.10 & 17.658 & 0.062 & 11.60 & 5.962 & 6.78 & 40.422 & 4.000 & 12.96 & 0.473 & 11.00 & 5.581 \\
\hline & 0.250 & 11.82 & 0.936 & 7.98 & 7.469 & 0.062 & 11.64 & 23.073 & 6.38 & 147.206 & 4.000 & 12.97 & 0.187 & 12.02 & 2.248 \\
\hline & 0.250 & 11.87 & 1.784 & 7.97 & 14.218 & 0.062 & 11.64 & 14.120 & 6.57 & 92.768 & 4.000 & 13.12 & 0.371 & 12.07 & 4.478 \\
\hline & 0.250 & 11.93 & 2.535 & 7.75 & 19.646 & 0.062 & 11.82 & 16.463 & 6.59 & 108.491 & 4.000 & 13.21 & 0.368 & 12.10 & 4.453 \\
\hline & 0.250 & 11.94 & 1.457 & 7.77 & 11.321 & 0.062 & 11.87 & 11.785 & 6.49 & 76.485 & 4.000 & 13.28 & 0.185 & 12.06 & 2.231 \\
\hline & 0.250 & 12.08 & 1.213 & 7.17 & 8.697 & 0.062 & 11.93 & 21.052 & 6.18 & 130.101 & 4.000 & 13.28 & 0.046 & 12.16 & 0.559 \\
\hline & 0.250 & 12.10 & 1.329 & 8.19 & 10.885 & 0.062 & 11.94 & 14.768 & 6.09 & 89.937 & 4.000 & 13.39 & 0.091 & 12.43 & 1.131 \\
\hline & 0.250 & 12.19 & 2.164 & 8.28 & 17.918 & 0.062 & 12.08 & 30.330 & 5.26 & 159.536 & 4.000 & 13.40 & 0.437 & 12.26 & 5.358 \\
\hline & 0.250 & 12.26 & 1.102 & 8.09 & 8.915 & 0.062 & 12.10 & 18.311 & 6.50 & 119.021 & 4.000 & 13.46 & 0.425 & 12.26 & 5.210 \\
\hline & 0.250 & 12.29 & 1.203 & 8.18 & 9.841 & 0.062 & 12.19 & 10.664 & 6.62 & 70.596 & 4.000 & 13.58 & 0.135 & 12.49 & 1.686 \\
\hline & 0.250 & 12.41 & 1.232 & 7.37 & 9.080 & 0.062 & 12.26 & 23.221 & 6.60 & 153.259 & 4.000 & 13.62 & 0.392 & 12.44 & 4.876 \\
\hline 0 & 0.250 & 12.41 & 2.330 & 7.94 & 18.500 & 0.062 & 12.29 & 29.952 & 6.50 & 194.688 & 4.000 & 13.62 & 0.180 & 12.57 & 2.263 \\
\hline & 0.250 & 12.43 & 1.789 & 8.54 & 15.278 & 0.062 & 12.41 & 38.471 & 5.27 & 202.742 & 4.000 & 13.66 & 0.132 & 12.80 & 1.690 \\
\hline & 0.250 & 12.52 & 1.551 & 8.83 & 13.695 & 0.062 & 12.41 & 11.911 & 6.27 & 74.682 & 4.000 & 13.66 & 0.224 & 12.43 & 2.784 \\
\hline & 0.250 & 12.56 & 2.865 & 8.65 & 24.782 & 0.062 & 12.43 & 18.470 & 6.93 & 127.997 & 4.000 & 13.80 & 0.356 & 12.54 & 4.464 \\
\hline & 0.250 & 12.56 & 1.854 & 8.59 & 15.926 & 0.062 & 12.52 & 21.478 & 7.56 & 162.374 & 4.000 & 13.81 & 0.449 & 12.77 & 5.632 \\
\hline & 0.250 & 12.66 & 3.689 & 8.84 & 32.611 & 0.062 & 12.56 & 11.635 & 7.88 & 91.684 & 4.000 & 13.84 & 0.310 & 12.49 & 3.872 \\
\hline & 0.250 & 12.68 & 1.896 & 8.96 & 16.988 & 0.062 & 12.56 & 18.713 & 7.28 & 1.36 .231 & 4.000 & 13.84 & 0.462 & 12.69 & 5.863 \\
\hline & 0.125 & 9.60 & 1.800 & 5.99 & 10.782 & 0.062 & 12.66 & 14.904 & 7.27 & 105.352 & 4.000 & 13.93 & 0.266 & 12.74 & 3.389 \\
\hline & 0.125 & 9.78 & 1.477 & 5.70 & 8.419 & 0.062 & 12.68 & 29.162 & 7.45 & 217.257 & 4.000 & 13.96 & 0.308 & 12.81 & 3.945 \\
\hline & 0.125 & 10.19 & 2.000 & 6.48 & 12.960 & 0.031 & 9.60 & 48.400 & 4.85 & 234.740 & 4.000 & 14.03 & 0.285 & 12.39 & 3.531 \\
\hline & 0.125 & 10.73 & 2.153 & 6.65 & 14.317 & 0.031 & 9.78 & 39.550 & 4.38 & 173.229 & 4.000 & 14.03 & 0.176 & 12.74 & 2.242 \\
\hline & 0.125 & 11.07 & 4.889 & 7.75 & 37.890 & 0.031 & 10.19 & 43.110 & 5.30 & 228.483 & 4.000 & 14.05 & 0.217 & 13.00 & 2.821 \\
\hline & 0.125 & 11.17 & 2.544 & 6.84 & 17.401 & 0.031 & 10.73 & 60.320 & 5.36 & 323.315 & 2.000 & 12.70 & 0.154 & 10.94 & 1.685 \\
\hline & 0.125 & 11.21 & 3.861 & 6.76 & 26.100 & 0.031 & 11.07 & 70.210 & 6.70 & 470.407 & 2.000 & 12.75 & 0.240 & 10.88 & 2.611 \\
\hline & 0.125 & 11.31 & 5.056 & 6.44 & 32.561 & 0.031 & 11.17 & 53.360 & 5.47 & 291.879 & 2.000 & 12.77 & 0.334 & 10.85 & 3.624 \\
\hline & 0.125 & 11.60 & 2.141 & 7.43 & 15.908 & 0.031 & 11.21 & 56.030 & 5.36 & 300.321 & 2.000 & 12.84 & 0.540 & 11.15 & 6.021 \\
\hline & 0.125 & 11.64 & 5.527 & 7.11 & 39.297 & 0.031 & 11.31 & 135.820 & 4.90 & 665.518 & 2.000 & 12.85 & 0.374 & 10.95 & 4.095 \\
\hline & 0.125 & 11.64 & 4.737 & 7.29 & 34.533 & 0.031 & 11.60 & 30.270 & 6.12 & 185.252 & 2.000 & 12.90 & 0.253 & 10.95 & 2.770 \\
\hline & 0.125 & 11.82 & 2.978 & 7.29 & 21.710 & 0.031 & 11.64 & 103.460 & 5.67 & 586.618 & 2.000 & 12.96 & 0.714 & 10.99 & 7.847 \\
\hline & 0.125 & 11.87 & 5.040 & 7.24 & 36.490 & 0.031 & 11.64 & 49.360 & 5.91 & 291.718 & 2.000 & 12.97 & 0.255 & 11.04 & 2.815 \\
\hline & 0.125 & 11.93 & 4.839 & 6.96 & 33.679 & 0.031 & 11.82 & 77.290 & 5.85 & 452.146 & 2.000 & 13.12 & 0.546 & 11.35 & 6.197 \\
\hline & 0.125 & 11.94 & 4.462 & 6.92 & 30.877 & 0.031 & 11.87 & 32.360 & 5.77 & 186.717 & 2.000 & 13.21 & 0.639 & 11.45 & 7.317 \\
\hline & 0.125 & 12.08 & 4.312 & 6.25 & 26.950 & 0.031 & 11.93 & 84.880 & 5.39 & 457.503 & 2.000 & 13.28 & 0.397 & 11.25 & 4.466 \\
\hline & 0.125 & 12.10 & 5.444 & 7.27 & 39.578 & 0.031 & 11.94 & 71.410 & 5.33 & 380.615 & 2.000 & 13.28 & 0.103 & 11.15 & 1.148 \\
\hline & 0.125 & 12.19 & 4.654 & 7.42 & 34.533 & 0.031 & 12.08 & 121.470 & 4.41 & 535.683 & 2.000 & 13.39 & 0.292 & 11.49 & 3.355 \\
\hline & 0.125 & 12.26 & 4.875 & 7.37 & 35.929 & 0.031 & 12.10 & 78.240 & 5.74 & 449.098 & 2.000 & 13.40 & 0.819 & 11.63 & 9.525 \\
\hline & 0.125 & 12.29 & 4.973 & 7.27 & 36.154 & 0.031 & 12.19 & 43.640 & 5.91 & 257.912 & 2.000 & 13.46 & 0.554 & 11.45 & 6.343 \\
\hline & 0.125 & 12.41 & 6.562 & 6.33 & 41.537 & 0.031 & 12.26 & 99.180 & 5.83 & 578.219 & 2.000 & 13.58 & 0.260 & 11.74 & 3.052 \\
\hline & 0.125 & 12.41 & 5.608 & 7.11 & 39.873 & 0.031 & 12.29 & 107.900 & 5.72 & 617.188 & 2.000 & 13.62 & 0.586 & 11.45 & 6.710 \\
\hline
\end{tabular}




\begin{tabular}{|c|c|c|c|c|c|c|c|c|c|c|c|c|c|c|}
\hline $\begin{array}{l}\text { Height } \\
(z)\end{array}$ & wind & $\begin{array}{l}\text { Mass } \\
\text { Conc }\end{array}$ & $\begin{array}{l}\text { Wind } \\
\text { e } 2 \mathrm{~m}\end{array}$ & $\begin{array}{l}\text { Mass } \\
\text { Flux }\end{array}$ & $\begin{array}{l}\text { He ight. } \\
(z)\end{array}$ & $e^{\text {Nind }} 10 \mathrm{~m}$ & $\begin{array}{l}\text { Mass } \\
\text { Conc }\end{array}$ & $\begin{array}{l}\text { Wind } \\
\text { e } z \mathrm{~m}\end{array}$ & $\begin{array}{l}\text { Mass } \\
\text { Flux }\end{array}$ & $\begin{array}{l}\text { teignt } \\
(z)\end{array}$ & $\begin{array}{l}\text { Wind } \\
\text { e 10 } 10\end{array}$ & $\begin{array}{l}\text { Mass } \\
\text { bon: }\end{array}$ & $\begin{array}{l}\text { Wind } \\
\text { e } z \text { n }\end{array}$ & $\begin{array}{l}\text { Muss } \\
\text { Fiux }\end{array}$ \\
\hline 2.000 & 13.62 & 0.288 & 11.91 & 3.430 & 0.500 & 13.40 & 2.041 & 9.82 & 20.0413 & 0.125 & 13.21 & 12.490 & 0.35 & 104.416 \\
\hline 2.000 & 13.66 & 0.327 & 11.93 & 3.901 & 0.500 & 13.46 & 1.877 & 9.39 & 18.564 & 0.125 & 13.28 & 7.755 & 8.90 & 69.019 \\
\hline 2.000 & 13.66 & 0.360 & 11.75 & 4.230 & 0.500 & 13.58 & 0.976 & 9.88 & 9.643 & 0.125 & 13.28 & 9.760 & 7.53 & 65.963 \\
\hline 2.000 & 13.80 & 0.717 & 11.84 & 8.489 & 0.500 & 13.62 & 2.088 & 9.75 & 20.358 & 0.125 & 13.39 & 5.628 & 8.3 .8 & 47.163 \\
\hline 2.000 & 13.81 & 0.660 & 12.01 & $7.92 \%$ & 0.500 & 13.62 & 0.672 & 9.88 & 6.639 & 0.125 & 13.40 & 7.729 & 8.28 & 63.996 \\
\hline 2.000 & 13.84 & 0.715 & 11.84 & 8.466 & 0.500 & 13.66 & 2.117 & 10.50 & 22.229 & 0.125 & 13.46 & 9.380 & 8.11 & 76.072 \\
\hline 2.000 & 13.84 & 0.612 & 11.76 & 7.197 & 0.500 & 13.66 & 1.177 & 10.05 & 11.829 & 0.125 & 13.58 & 6.769 & 8.36 & 56.589 \\
\hline 2.000 & 13.93 & 0.477 & 11.77 & 5.614 & 0.500 & 13.80 & 1.800 & 9.94 & 17.892 & 0.125 & 13.62 & 9.356 & 7.86 & 73.538 \\
\hline 2.000 & 13.96 & 0.664 & 11.79 & 7.829 & 0.500 & $: 3.81$ & 2.187 & 10.23 & 22.373 & 0.125 & 13.62 & 7.177 & 8.37 & 50.071 \\
\hline 2.000 & 14.03 & 0.567 & 11.98 & 6.793 & 0.500 & 13.84 & 2.244 & 10.13 & 22.732 & 0.125 & 13.66 & 8.789 & 9.20 & 80.859 \\
\hline 2.000 & 14.03 & 0.332 & 11.86 & 3.938 & 0.500 & 13.84 & 2.397 & 9.93 & 23.802 & 0.125 & 13.66 & 5.000 & 8.34 & 41.700 \\
\hline 2.000 & 14.05 & 0.277 & 12.35 & 3.421 & 0.500 & 13.93 & 1.648 & 9.96 & 16.414 & 0.125 & 13.80 & 9.026 & 8.21 & 74.103 \\
\hline 1.000 & 12.70 & 0.278 & 9.85 & $2: 738$ & 0.500 & 13.96 & 2.582 & 10.19 & 26.311 & 0.125 & 13.81 & 13.125 & 8.64 & 113.400 \\
\hline $1.000^{\circ}$ & 12.75 & 0.369 & 10.02 & 3.697 & 0.500 & 14.03 & 2.207 & 10.14 & 22.379 & 0.125 & 13.84 & 11.734 & 8.23 & 96.571 \\
\hline 1.000 & 12.77 & 0.638 & 10.07 & 6.425 & 0.500 & 14.03 & 1.700 & 9.94 & 16.898 & 0.125 & 13.84 & 10.122 & 8.12 & $82 .: 91$ \\
\hline 1.000 & 12.84 & 0.867 & 10.29 & 8.921 & 0.500 & 14.05 & 0.640 & 10.33 & 6.611 & 0.125 & 13.93 & 9.041 & 8.01 & 72.418 \\
\hline 1.000 & 12.85 & 0.548 & 10.26 & 5.622 & 0.250 & 12.70 & 1.451 & 8.53 & 12.377 & 0.125 & 13.96 & 15.599 & 8.17 & .127 .444 \\
\hline 1.000 & 12.90 & 0.408 & 10.29 & 4.198 & 0.250 & 12.75 & 2.145 & 8.52 & 18.275 & 0.125 & 14.03 & 11.540 & 8.20 & 34.628 \\
\hline 1.000 & 12.96 & 1.764 & 10.31 & 18.187 & 0.250 & 12.77 & 2.859 & 9.30 & 26.589 & 0.125 & 14.03 & 10.920 & 3.02 & 87.578 \\
\hline 1.000 & 12.97 & 0.382 & 10.28 & 3.927 & 0.250 & 12.84 & 2.405 & 8.95 & 21.525 & 0.125 & 14.05 & 2.394 & 8.91 & 21.331 \\
\hline 1.000 & 13.12 & 1.119 & 10.56 & 11.817 & 0.250 & 12.85 & 2.2914 & 8.69 & 19.935 & 0.062 & 12.70 & 28.393 & 7.00 & 198.751 \\
\hline 1.000 & 13.21 & 1.316 & 10.67 & 14.042 & 0.250 & 12.90 & 1.922 & 8.85 & 17.010 & 0.062 & 12.75 & 19.600 & 6.97 & 136.612 \\
\hline 1.000 & 13.28 & 0.672 & 10.43 & 7.009 & 0.250 & 12.96 & 5.284 & 8.42 & 44.491 & 0.062 & 12.77 & 34.603 & 6.96 & 240.837 \\
\hline 1.000 & 13.28 & 0.356 & 10.16 & 3.617 & 0.250 & 12.97 & 1.340 & 8.80 & 11.792 & 0.062 & 12.84 & 20.465 & 7.37 & 150.827 \\
\hline 1.000 & 13.39 & 0.574 & 10.67 & 6.125 & 0.250 & 13.12 & 4.309 & 9.00 & 38.781 & 0.062 & 12.85 & 22.922 & 7.07 & 162.059 \\
\hline 1.000 & 13.40 & 1.112 & 10.37 & 11.531 & 0.250 & 13.21 & 3.689 & 9.22 & 34.013 & 0.062 & 12.90 & 17.426 & 7.20 & 125.467 \\
\hline 1.000 & 13.46 & 1.057 & 10.62 & 11.225 & 0.250 & 13.28 & 2.634 & 9.76 & 25.708 & 0.062 & 12.96 & 28.439 & 6.83 & 194.238 \\
\hline 1.000 & 13.58 & 0.497 & 10.80 & 5.368 & 0.250 & 13.28 & 2.095 & 8.56 & 17.933 & 0.062 & 12.97 & 19.711 & 7.16 & 141.131 \\
\hline 1.000 & 13.62 & 1.024 & 10.63 & 10.885 & 0.250 & 13.39 & 1.528 & 9.22 & 14.088 & 0.062 & 13.12 & 32.522 & 7.44 & 241.964 \\
\hline 1.000 & 13.62 & 0.465 & 10.86 & 5.050 & 0.250 & 13.40 & 3.409 & 9.12 & 31.090 & 0.062 & 13.21 & 25.114 & 7.48 & 187.853 \\
\hline 1.000 & 13.66 & 0.695 & 11.35 & 7.888 & 0.250 & 13.46 & 4.159 & 8.99 & 37.389 & 0.062 & 13.28 & 26.074 & 7.03 & $\begin{array}{r}13.300 \\
0\end{array}$ \\
\hline 1.000 & 13.66 & 0.595 & 10.90 & 0.485 & 0.250 & 13.58 & 2.676 & 9.29 & 24.860 & 0.062 & 13.28 & 25.737 & 6.62 & 170.379 \\
\hline 1.000 & 13.80 & 1.034 & 10.92 & $: 1.291$ & 0.250 & 13.62 & 4.310 & 8.69 & 37.454 & 0.062 & 13.39 & 14.252 & 7.59 & 108.173 \\
\hline 1.000 & 13.81 & 1.215 & 10.87 & 13.207 & 0.250 & 13.62 & 1.770 & 8.74 & 15.470 & 0.062 & 13.40 & 12.829 & 8.39 & 107.635 \\
\hline 1.000 & 13.84 & 1.341 & 10.83 & 14.523 & 0.250 & 13.66 & 4.537 & 9.99 & 45.325 & 0.062 & 13.46 & 25.367 & 7.05 & 170.837 \\
\hline 1.000 & 13.84 & 1.037 & 10.70 & 11.096 & 0.250 & 13.66 & 2.538 & 9.30 & 23.603 & 0.062 & 13.58 & 36.229 & 7.54 & 273.167 \\
\hline 1.000 & 13.93 & 0.986 & 10.63 & 10.481 & 0.250 & 13.80 & 3.459 & 9.07 & 31.373 & 0.062 & 13.62 & 24.394 & 6.97 & 170.026 \\
\hline 1.000 & 13.96 & 1.182 & 10.76 & 12.718 & 0.250 & 13.81 & 4.277 & 9.57 & 40.931 & 0.062 & 13.62 & 17.794 & 7.88 & 140.217 \\
\hline 1.000 & 14.03 & 1.100 & 11.26 & 12.386 & 0.250 & 13.84 & 4.740 & 9.47 & 44.888 & 0.062 & 13.66 & 28.907 & 8.49 & 245.420 \\
\hline 1.000 & 14.03 & 0.878 & 10.87 & 9.544 & 0.250 & 13.84 & 4.979 & 9.08 & 45.209 & 0.062 & 13.66 & 11.041 & 7.39 & 81.593 \\
\hline 1.000 & 14.05 & 0.445 & 11.34 & 5.046 & 0.250 & 13.93 & 3.388 & 9.05 & 30.661 & 0.062 & 13.80 & 13.397 & 8.02 & 107.444 \\
\hline 0.500 & 12.70 & 0.665 & 9.30 & 6.184 & 0.250 & 13.96 & 5.873 & 9.03 & 53.033 & 0.062 & 13.81 & 27.495 & 7.82 & 215.011 \\
\hline 0.500 & 12.75 & 0.802 & 9.47 & 7.595 & 0.250 & 14.03 & 4.986 & 9.45 & 47.118 & $0: 062$ & 13.84 & 24.240 & 7.05 & 170.892 \\
\hline 0.500 & 12.77 & 1.262 & 10.07 & 12.708 & 0.250 & 14.03 & 4.260 & 8.95 & 38.127 & 0.062 & 13.84 & 25.240 & 7.21 & 181.980 \\
\hline 0.500 & 12.84 & 1.285 & 9.53 & 12.246 & 0.250 & 14.05 & 1.098 & 9.45 & 10.376 & 0.062 & 13.93 & 32.874 & 7.03 & 231.104 \\
\hline 0.500 & 12.85 & 0.951 & 9.38 & 8.920 & 0.125 & 12.70 & 5.592 & 7.73 & 43.226 & 0.062 & 13.96 & 37.710 & 7.25 & 273.397 \\
\hline 0.500 & 12.90 & 0.736 & 9.49 & 6.985 & 0.125 & 12.75 & 6.753 & 7.76 & 52.403 & 0.062 & 14.03 & 35.280 & 6.90 & 243.432 \\
\hline 0.500 & 12.96 & 2.405 & 9.36 & 22.511 & 0.125 & 12.77 & 7.352 & 8.39 & 61.683 & 0.062 & 14.03 & 41.150 & 6.60 & 271.590 \\
\hline 0.500 & 12.97 & 0.706 & 9.54 & 6.735 & 0.125 & 12.84 & 7.835 & 8.12 & 63.620 & 0.062 & 14.05 & 5.968 & 8.34 & 49.773 \\
\hline 0.500 & 13.12 & 1.552 & 9.74 & 15.116 & 0.125 & 12.85 & 6.667 & 7.86 & 52.403 & 0.031 & 12.70 & 116.750 & 6.15 & 718.012 \\
\hline 0.500 & 13.21 & 1.982 & 10.00 & 19.820 & 0.125 & 12.90 & 5.628 & 7.98 & 44.911 & 0.031 & 12.75 & 87.070 & 6.19 & 538.963 \\
\hline 0.500 & 13.28 & 1.171 & 9.59 & 11.230 & 0.125 & 12.96 & 10.407 & 7.66 & 79.718 & 0.031 & 12.77 & 167.400 & 7.05 & 1180.170 \\
\hline 0.500 & 13.28 & 0.751 & 9.26 & 6.954 & 0.125 & 12.97 & 4.731 & 7.95 & 37.611 & 0.031 & 12.84 & 92.740 & 6.63 & 614.866 \\
\hline 0.500 & 13.39 & 0.845 & 9.99 & 8.442 & 0.125 & 13.12 & 10.517 & 8.22 & 86.450 & 0.031 & 12.85 & 108.640 & 6.28 & 682.259 \\
\hline
\end{tabular}




\begin{tabular}{|c|c|c|c|c|c|c|c|c|c|c|c|c|c|c|}
\hline $\begin{array}{l}\text { Feight } \\
(z)\end{array}$ & $\begin{array}{l}\text { Wind } \\
\text { (e) } 10 \mathrm{~m}\end{array}$ & $\begin{array}{l}\text { Mass } \\
\text { Conc }\end{array}$ & $\begin{array}{l}\text { Wind } \\
e z \mathrm{~m}\end{array}$ & $\begin{array}{l}\text { Mass } \\
\text { Flux }\end{array}$ & $\begin{array}{l}\text { Height } \\
(z)\end{array}$ & $\begin{array}{l}\text { Wind } \\
e 10 \mathrm{~m}\end{array}$ & $\begin{array}{l}\text { Mass } \\
\text { Conc }\end{array}$ & $\begin{array}{l}\text { Wind } \\
\text { e z m }\end{array}$ & $\begin{array}{l}\text { Mase } \\
\text { Flux }\end{array}$ & $\begin{array}{l}\text { Height } \\
(z)\end{array}$ & $\begin{array}{l}\text { Wind } \\
e 10 \mathrm{~m}\end{array}$ & $\begin{array}{l}\text { Mass } \\
\text { Cono }\end{array}$ & $\begin{array}{l}\text { Wind } \\
\text { è } \mathrm{zm}\end{array}$ & $\begin{array}{l}\text { Mass } \\
\text { Fl i. }\end{array}$ \\
\hline 0.031 & 12.90 & 124.930 & 6.43 & 803.300 & 2.000 & 14.07 & 0.123 & 12.04 & 1.481 & 1.000 & 16.13 & 0.659 & 12.67 & 8.350 \\
\hline 0.031 & 12.96 & 105.490 & 5.99 & 631.885 & 2.000 & 14.17 & 0.509 & 12.42 & 0.322 & 1.000 & 16.51 & 1.925 & 13.37 & 25.737 \\
\hline 0.031 & 12.97 & 65.920 & 6.37 & 419.910 & 2.000 & 14.23 & 0.610 & 12.27 & 7.485 & 1.000 & $i 6.54$ & 1.621 & 12.87 & 20.862 \\
\hline 0.031 & 13.12 & 90.900 & 6.67 & 606.303 & 2.000 & 14.29 & 0.556 & 11.98 & 6.661 & 1.000 & 16.56 & 2.771 & 12.33 & 34.166 \\
\hline 0.031 & 13.21 & 73.970 & 6.83 & 505.215 & 2.000 & 14.31 & 0.473 & 12.00 & 5.676 & 1.000 & 15.58 & 1.979 & 13.27 & 26.261 \\
\hline 0.031 & 13.28 & 87.160 & 6.85 & 597.046 & 2.000 & 14.31 & 0.112 & 12.42 & 1.391 & 1.000 & 16.78 & 2.507 & 12.55 & 31.463 \\
\hline 0.031 & 13.28 & 11.3 .800 & 5.71 & 649.798 & 2.000 & 14.41 & 0.758 & 12.38 & 9.384 & 0.500 & 14.07 & 1.304 & 10.44 & 13.614 \\
\hline 0.031 & 13.39 & 52.590 & 6.80 & 357.612 & 2.000 & 14.45 & 0.687 & 12.44 & 8.546 & 0.500 & 14.17 & 1.240 & 10.38 & 12.871 \\
\hline 0.031 & 13.40 & 47.330 & 6.66 & 315.218 & 2.000 & 14.57 & 0.675 & 12.47 & 8.417 & 0.500 & 14.23 & 2.371 & 10.56 & 25.275 \\
\hline 0.031 & 13.46 & 82.750 & 6.35 & 525.463 & 2.000 & 14.59 & 0.714 & 12.44 & 8.882 & 0.500 & 14.29 & 2.845 & 10.26 & 29.190 \\
\hline 0.031 & 13.58 & 123.080 & 6.71 & 825.867 & 2.000 & 14.66 & 0.356 & 12.52 & 4.457 & 0.500 & 14.31 & 2.761 & 10.09 & 27.858 \\
\hline 0.031 & 13.62 & 111.340 & 6.04 & 672.494 & 2.000 & 14.86 & 1.087 & 12.85 & 13.968 & 0.500 & 14.31 & 1.338 & 10.96 & 14.664 \\
\hline 0.031 & 13.62 & 77.680 & 6.70 & 520.456 & 2.000 & 14.89 & 0.351 & 12.85 & 4.510 & 0.500 & 14.41 & 2.052 & 10.70 & 21.956 \\
\hline 0.031 & 13.66 & 110.270 & 7.79 & 859.003 & 2.000 & 15.01 & 0.702 & 12.85 & 9.021 & 0.500 & 14.45 & 3.255 & 10.16 & 33.071 \\
\hline 0.031 & 13.66 & 43.360 & 6.66 & 288.778 & 2.000 & 15.02 & 0.561 & 12.83 & 7.198 & 0.500 & 14.57 & 2.319 & 10.73 & 24.883 \\
\hline 0.031 & 13.80 & 58.500 & 6.44 & 376.740 & 2.000 & 15.29 & 0.833 & 12.74 & 10.612 & 0.500 & 14.59 & 2.488 & 10.76 & 26.771 \\
\hline 0.031 & 13.81 & 140.750 & 7.00 & 985.250 & 2.000 & 15.57 & 1.292 & $13.06^{\circ}$ & 16.874 & 0.500 & 14.66 & 1.514 & 10.87 & 16.457 \\
\hline 0.031 & 13.84 & 90.430 & 6.45 & 583.273 & 2.000 & 15.60 & 1.011 & 13.28 & 13.426 & 0.500 & 14.86 & 4.003 & 11.22 & 44.914 \\
\hline 0.031 & 13.84 & 98.640 & 6.31 & 622.418 & 2.000 & 15.69 & 1.170 & 13.44 & 15.725 & 0.500 & 14.89 & 1.330 & 10.95 & 14.563 \\
\hline 0.031 & 13.93 & 131.130 & 6.14 & 805.138 & 2.000 & 15.77 & 0.746 & 13.78 & 10.280 & 0.500 & 15.01 & 2.217 & 11.02 & 24.431 \\
\hline 0.031 & 13.96 & 165.580 & 6.34 & 1049.777 & 2.000 & 15.86 & 0.909 & 13.46 & 12.235 & 0.500 & 15.02 & 1.724 & 11.13 & 19.188 \\
\hline 0.031 & 14.03 & 135.830 & 6.35 & 862.520 & 2.000 & 15.91 & 0.656 & 13.64 & 8.948 & 0.500 & 15.29 & 2.839 & 10.90 & 30.945 \\
\hline 0.031 & 14.03 & 121.740 & 6.12 & 745.049 & 2.000 & 16.01 & 0.830 & 13.44 & 11.155 & 0.500 & 15.57 & 5.386 & 10.78 & 58.061 \\
\hline 0.031 & 14.05 & 17.660 & 7.20 & 127.152 & 2.000 & 16.02 & $\therefore .315$ & 13.83 & 18.186 & 0.500 & 15.60 & 3.904 & 11.31 & 44.154 \\
\hline 4.000 & 14.07 & 0.058 & 13.05 & 0.757 & 2.000 & 16.13 & 0.285 & 14.07 & 4.010 & 0.500 & 15.69 & 3.316 & 11.47 & 38.035 \\
\hline 4.000 & 14.17 & 0.129 & 13.11 & 1.691 & 2.000 & 16.51 & 0.861 & 14.30 & 12.312 & 0.500 & 15.77 & 2.409 & 11.63 & 28.017 \\
\hline 4.000 & 14.23 & 0.371 & 13.11 & 4.864 & 2.000 & 16.54 & 1.088 & 14.08 & 15.319 & 0.500 & 15.86 & 3.478 & 11.70 & 40.693 \\
\hline 4.000 & 14.29 & 0.344 & 13.08 & 4.500 & 2.000 & 16.56 & 1.224 & 13.78 & 16.867 & 0.500 & 15.91 & 2.385 & 11.60 & 27.666 \\
\hline 4.000 & 14.31 & 0.304 & 12.73 & 3.870 & 2.000 & 16.58 & 1.103 & 14.36 & 15.839 & 0.500 & 16.01 & 3.253 & 11.54 & 37.540 \\
\hline 4.000 & 14.31 & 0.063 & 13.31 & 0.839 & 2.000 & 16.78 & 1.382 & 14.05 & 19.417 & 0.500 & 16.02 & 4.880 & 11.72 & 57.194 \\
\hline 4.000 & 14.41 & 0.480 & 13.14 & 6.307 & 1.000 & 14.07 & 0.330 & 11.36 & 3.749 & 0.500 & 16.13 & 1.717 & 11.72 & 0.123 \\
\hline 4.000 & 14.45 & 0.340 & 13.18 & 4.481 & 1.000 & 14.17 & 0.896 & 11.28 & 10.107 & 0.500 & 16.51 & 3.869 & 12.44 & 48.130 \\
\hline 4.000 & 14.57 & 0.420 & 13.31 & 5.590 & 1.000 & 14.23 & 1.113 & 11.43 & 12.722 & 0.500 & 16.54 & 3.458 & 11.80 & 40.804 \\
\hline 4.000 & 14.59 & 0.417 & 13.60 & 5.671 & 1.000 & 14.29 & 1.054 & 11.23 & 11.836 & 0.500 & 16.56 & 6.029 & 11.23 & 67.706 \\
\hline 4.000 & 14.66 & 0.194 & 13.57 & 2.633 & 1.000 & 14.31 & 1.154 & 10.63 & 12.267 & 0.500 & 16.58 & 4.281 & 12.19 & 52.185 \\
\hline 4.000 & 14.86 & 0.572 & 13.85 & 7.922 & 1.000 & 14.31 & 0.432 & 11.70 & 5.054 & 0.500 & 16.78 & 6.382 & 12.55 & 80.094 \\
\hline 4.000 & 14.89 & 0.205 & 13.61 & 2.790 & 1.000 & 14.41 & 1.142 & 11.40 & 13.019 & 0.250 & 14.07 & 3.793 & 9.71 & 36.830 \\
\hline 4.000 & 15.01 & 0.286 & 13.60 & 3.890 & 1.000 & 14.45 & 1.342 & 11.30 & 15.165 & 0.250 & 14.17 & 1.765 & 9.49 & 16.750 \\
\hline 4.000 & 15.02 & 0.262 & 13.91 & 3.644 & 1.000 & 14.57 & 1.117 & 11.56 & 12.913 & 0.250 & 14.23 & 5.572 & 9.63 & 53.658 \\
\hline 4.000 & 15.29 & 0.566 & 13.91 & 7.873 & 1.000 & 14.59 & 1.344 & 11.60 & 15.590 & 0.250 & 14.29 & 7.107 & 9.51 & 67.588 \\
\hline 4.000 & 15.57 & 0.675 & 14.20 & 9.585 & 1.000 & 14.66 & 0.541 & 11.80 & 6.384 & 0.250 & 14.31 & 5.228 & 8.42 & 44.020 \\
\hline 4.000 & 15.60 & 0.628 & 14.28 & 8.968 & 1.000 & 14.86 & 2.095 & 11.95 & 25.035 & 0.250 & 14.31 & 4.185 & 10.11 & 42.310 \\
\hline 4.000 & 15.69 & 0.545 & 14.45 & 7.875 & 1.000 & 14.89 & 0.614 & 11.97 & 7.350 & 0.250 & 14.41 & 4.167 & 9.88 & 41.170 \\
\hline 4.000 & 15.77 & 0.387 & 14.40 & 5.573 & 1.000 & 15.01 & 1.185 & 11.84 & 14.030 & 0.250 & 14.45 & 6.802 & 9.25 & 62.918 \\
\hline 4.000 & 15.86 & 0.463 & 14.60 & 6.760 & 1.000 & 15.02 & 0.819 & 12.00 & 9.828 & 0.250 & 14.57 & 5.360 & 9.76 & 52.314 \\
\hline 4.000 & 15.91 & 0.307 & 14.81 & 4.547 & 1.000 & 15.29 & 1.433 & 11.58 & 16.594 & 0.250 & 14.59 & 5.411 & 10.09 & 54.597 \\
\hline 4.000 & 16.01 & 0.538 & 14.61 & 7.860 & 1.000 & 15.57 & 2.447 & 11.77 & 28.801 & 0.250 & 14.66 & 4.186 & 10.02 & 41.944 \\
\hline 4.000 & 16.02 & 0.727 & 14.57 & 10.592 & 1.000 & 15.60 & 1.774 & 12.47 & 22.122 & 0.250 & 14.86 & 8.167 & 10.45 & 85.345 \\
\hline 4.000 & 16.13 & 0.114 & 14.65 & 1.670 & 1.000 & 15.69 & 1.889 & 12.42 & 23.461 & 0.250 & 14.89 & 3.175 & 10.07 & 31.972 \\
\hline 4.000 & 16.51 & 0.367 & 15.34 & 5.630 & 1.000 & 15.77 & 1.425 & 12.49 & 17.798 & 0.250 & 15.01 & 4.975 & 10.05 & 49.999 \\
\hline 4.000 & 16.54 & 0.410 & 14.89 & 6.105 & 1.000 & 15.86 & 1.870 & 12.47 & 23.319 & 0.250 & 15.02 & 4.259 & 10.25 & 43.655 \\
\hline 4.000 & 16.56 & 0.600 & 15.10 & 9.060 & 1.000 & 15.91 & 1.368 & 12.72 & 17.401 & 0.250 & 15.29 & 6.186 & 9.82 & 60.747 \\
\hline 4.000 & 16.58 & 0.540 & 15.17 & 8.192 & 1.000 & 16.01 & 1.667 & 12.32 & 20.537 & 0.250 & 15.57 & 10.778 & 9.86 & 106.271 \\
\hline 4.000 & 16.78 & 0.521 & 14.93 & 7.779 & 1.000 & 16.02 & 2.399 & 12.54 & 30.083 & 0.250 & 15.60 & 8.707 & 10.36 & 90.205 \\
\hline
\end{tabular}




\begin{tabular}{|c|c|c|c|c|c|c|c|c|c|c|c|c|c|c|}
\hline $\begin{array}{l}\text { Height } \\
(z)\end{array}$ & $e^{\text {Wind }}$ & $\begin{array}{l}\text { Mass } \\
\text { Conc }\end{array}$ & $\begin{array}{l}\text { Wind } \\
\text { e } z \mathrm{~m}\end{array}$ & $\begin{array}{l}\text { Mass } \\
\text { Flux }\end{array}$ & $\begin{array}{l}\text { Height } \\
(z)\end{array}$ & $\begin{array}{l}\text { Wind } \\
\text { e } 10 \mathrm{~m}\end{array}$ & $\begin{array}{l}\text { Mass } \\
\text { Conc }\end{array}$ & $\begin{array}{l}\text { Wind } \\
\text { o } z \mathrm{~m}\end{array}$ & $\begin{array}{l}\text { Mass } \\
\text { Flux }\end{array}$ & $\begin{array}{l}\text { Fe ight } \\
(z)\end{array}$ & $e^{\text {Wind }} 10 \mathrm{~m}$ & $\begin{array}{l}\text { Mass } \\
\text { Conc }\end{array}$ & $\begin{array}{l}\text { Wind } \\
\oplus \mathrm{zm}\end{array}$ & $\begin{array}{l}\text { Mass } \\
\text { Flux }\end{array}$ \\
\hline 0.250 & 15.69 & 7.030 & 10.60 & 74.518 & 0.062 & 14.89 & 25.728 & 8.25 & 212.256 & 4.000 & 17.25 & 0.353 & 15.95 & 5.630 \\
\hline 0.250 & 15.77 & 5.356 & 10.70 & 57.309 & 0.062 & 15.01 & 34.537 & 7.39 & 272.497 & 4.000 & 17.35 & 0.599 & 15.59 & 9.333 \\
\hline 0.250 & 15.86 & 7.228 & 11.02 & 79.653 & 0.062 & 15.02 & 26.918 & 8.42 & 226.650 & 4.000 & 17.48 & 0.884 & 15.59 & 13.782 \\
\hline 0.250 & 15.91 & 5.712 & 10.66 & 60.890 & 0.062 & 15.29 & 48.411 & 7.46 & 361.146 & 4.000 & 17.51 & 0.452 & 16.27 & 7.354 \\
\hline 0.250 & 16.01 & 7.235 & 10.56 & 76.402 & 0.062 & 15.57 & 60.898 & 7.55 & 459.780 & 4.000 & 17.51 & 0.657 & 15.82 & 10.394 \\
\hline 0.250 & 16.02 & 11.694 & 10.54 & 123.255 & 0.062 & 15.60 & 52.053 & 7.93 & 412.780 & 4.000 & 17.72 & 0.932 & 16.27 & 15.164 \\
\hline 0.250 & 16.13 & 5.113 & 10.78 & 55.118 & 0.062 & 15.69 & 61.942 & 8.61 & 533.321 & 4.000 & 17.76 & 0.967 & 16.40 & 15.859 \\
\hline 0.250 & 16.51 & 9.875 & 11.18 & 110.402 & 0.062 & 15.77 & 40.026 & 8.78 & 351.428 & 4.000 & 17.79 & 0.554 & 16.28 & 9.019 \\
\hline 0.250 & 16.54 & 14.088 & 10.41 & 146.656 & 0.062 & 15.86 & 30.528 & 8.46 & 258.267 & 4.000 & 17.92 & 0.837 & 16.08 & 13.459 \\
\hline 0.250 & 16.56 & 12.168 & 10.36 & 126.060 & 0.062 & 15.91 & 34.100 & 9.08 & 309.628 & 4.000 & 17.98 & 0.619 & 16.15 & 9.997 \\
\hline 0.250 & 16.58 & 10.398 & 11.23 & 116.770 & 0.062 & 16.01 & 50.718 & 8.01 & 406.251 & 4.000 & 18.02 & 0.823 & 16.04 & 13.201 \\
\hline 0.250 & 16.78 & 14.401 & 11.18 & 161.003 & 0.062 & 16.02 & 54.632 & 8.57 & 468.196 & 4.000 & 18.20 & 0.816 & 16.69 & 13.619 \\
\hline 0.125 & 14.07 & 10.288 & 8.84 & 90.946 & 0.062 & 16.13 & 65.261 & 8.74 & 570.381 & 4.000 & 18.21 & 1.071 & 17.02 & 18.228 \\
\hline 0.125 & 14.17 & 5.569 & 8.67 & 48.283 & 0.062 & 16.51 & 75.323 & 9.67 & 728.373 & 4.000 & 18.39 & 0.401 & 16.60 & 6.657 \\
\hline 0.125 & 14.23 & 14.213 & 8.90 & 126.496 & 0.062 & 16.54 & 66.142 & 8.31 & 549.640 & 4.000 & 18.42 & 0.736 & 16.72 & 12.306 \\
\hline 0.125 & 14.29 & 13.918 & 8.39 & 116.772 & 0.062 & 16.56 & 73.018 & 7.75 & 565.890 & 4.000 & 18.42 & 0.739 & 16.74 & 12.371 \\
\hline 0.125 & 14.31 & 10.128 & 7.65 & 77.479 & 0.062 & 16.58 & 53.338 & 9.22 & 491.776 & 4.000 & 18.57 & 0.881 & 16.79 & 14.792 \\
\hline 0.125 & 14.31 & 13.194 & 7.34 & 96.844 & 0.062 & 16.78 & 88.402 & 7.43 & 656.827 & 4.000 & 18.65 & 0.743 & 17.00 & 12.631 \\
\hline 0.125 & 14.41 & 9.548 & 8.82 & 84.213 & 0.031 & 14.07 & 146.110 & 7.19 & 1050.531 & 4.000 & 18.80 & 0.847 & 17.16 & 14.535 \\
\hline 0.125 & 14.45 & 12.079 & 8.46 & 102.188 & 0.031 & 14.17 & 43.750 & 6.93 & 303.187 & 4.000 & 19.11 & 0.963 & 17.36 & 16.718 \\
\hline 0.125 & 14.57 & 14.035 & 8.92 & 125.192 & 0.031 & 14.23 & 113.940 & 7.21 & 921.507 & 4.000 & 19.39 & 1.518 & 17.79 & 27.005 \\
\hline 0.125 & 14.59 & 15.254 & 9.09 & 138.659 & 0.031 & 14.29 & 111.840 & 6.52 & 729.197 & 4.000 & 19.46 & 0.636 & 17.63 & 11.213 \\
\hline 0.125 & 14.66 & 11.847 & 9.13 & 108.163 & 0.031 & 14.31 & 106.120 & 5.55 & 588.966 & 4.000 & 19.66 & 0.659 & 17.99 & 11.855 \\
\hline 0.125 & 14.86 & 20.950 & 9.54 & 199.863 & 0.031 & 14.31 & 153.350 & 7.27 & 11114.854 & 4.000 & 19.81 & 1.122 & 18.09 & 20.297 \\
\hline 0.125 & 14.89 & 7.101 & 9.17 & 65.116 & 0.031 & 14.41 & 83.500 & 7.05 & 588.675 & 2.000 & 16.91 & 1.383 & 14.47 & 20.012 \\
\hline 0.125 & 15.01 & 14.379 & 8.98 & 129.123 & 0.031 & 14.45 & 117.510 & 6.56 & 770.866 & 2.000 & 16.94 & 0.390 & 14.42 & 5.624 \\
\hline 0.125 & 15.02 & 11.064 & 9.32 & 103.116 & 0.031 & 14.57 & 112.850 & 7.02 & 792.207 & 2.000 & 17.04 & 2.304 & 14.78 & 34.053 \\
\hline 0.125 & 15.29 & 16.940 & 8.38 & 141.957 & 0.031 & 14.59 & 88.060 & 7.35 & 647.241 & 2.000 & 17.07 & 0.689 & 14.53 & 10.080 \\
\hline 0.125 & 15.57 & 22.391 & 8.65 & 193.682 & 0.031 & 14.66 & 172.720 & 7.38 & 1274.674 & 2.000 & 17.11 & 1.526 & 14.61 & 22.295 \\
\hline 0.125 & 15.60 & 17.021 & 9.40 & 159.997 & 0.031 & 14.86 & 96.540 & 7.85 & 757.839 & 2.000 & 17.20 & 1.408 & 14.56 & 20.500 \\
\hline 0.125 & 15.69 & 18.753 & 9.58 & 179.654 & 0.031 & 14.89 & 75.810 & 7.36 & 557.962 & 2.000 & 17.25 & 0.791 & 14.81 & 11.715 \\
\hline 0.125 & 15.77 & 14.064 & 9.74 & 136.983 & 0.031 & 15.01 & 144.120 & 7.07 & 1013.928 & 2.000 & 17.35 & 1.435 & 14.80 & $2 ! .238$ \\
\hline 0.125 & 15.86 & 13.482 & 9.66 & 130.236 & 0.031 & 15.02 & 95.010 & 7.51 & 713.525 & 2.000 & 17.48 & 1.455 & 14.80 & 21.534 \\
\hline 0.125 & 15.91 & 12.823 & 9.85 & 126.307 & 0.031 & 15.29 & 200.420 & 6.42 & 1286.696 & 2.000 & 17.51 & 0.927 & 14.93 & 1.3 .840 \\
\hline 0.125 & 16.01 & 19.907 & 9.25 & 184.140 & 0.031 & 15.57 & 163.190 & 6.45 & 1052.575 & 2.000 & 17.51 & 1.367 & 14.93 & 20.409 \\
\hline 0.125 & 16.02 & 24.001 & 9.59 & 230.170 & 0.031 & 15.60 & 183.730 & 7.44 & 1366.951 & 2.000 & 17.72 & 1.849 & 15.17 & 28.049 \\
\hline 0.125 & 16.13 & 23.492 & 9.75 & 229.047 & 0.031 & 15.69 & 155.780 & 7.64 & 1190.159 & 2.000 & 17.76 & 1.745 & 14.96 & 26.105 \\
\hline 0.125 & 16.51 & 28.784 & 10.61 & 305.398 & 0.031 & 15.77 & 15.099 & 7.83 & 118.225 & 2.000 & 17.79 & 1.271 & 15.09 & 19.179 \\
\hline 0.125 & 16.54 & 28.307 & 9.44 & 267.218 & 0.031 & 15.86 & 93.830 & 7.70 & 722.491 & 2.000 & 17.92 & 1.717 & 14.76 & 25.343 \\
\hline 0.125 & 16.56 & 27.035 & 8.95 & 241.963 & 0.031 & 15.91 & 130.260 & 7.93 & 1032.962 & 2.000 & 17.98 & 1.060 & 15.22 & 16.133 \\
\hline 0.125 & 16.58 & 21.000 & 10.23 & 214.830 & 0.031 & 16.01 & 203.420 & 7.11 & 1446.316 & 2.000 & 18.02 & 1.522 & 15.10 & 22.982 \\
\hline 0.125 & 16.78 & 36.904 & 9.98 & 368.302 & 0.031 & 16.02 & 169.310 & 7.56 & 1279.984 & 2.000 & 18.20 & 1.606 & 15.14 & 24.315 \\
\hline 0.062 & 14.07 & 41.532 & 8.02 & 333.087 & 0.031 & 16.13 & 227.170 & 7.77 & 1765.111 & 2.000 & 18.21 & 1.862 & 15.65 & 29.140 \\
\hline 0.062 & 14.17 & 14.971 & 8.05 & 120.517 & 0.031 & 16.51 & 249.410 & 8.74 & 2179.843 & 2.000 & 18.39 & 0.820 & 15.71 & 12.882 \\
\hline 0.062 & 14.23 & 46.120 & 8.09 & 373.111 & 0.031 & 16.54 & 219.010 & 7.19 & 1574.682 & 2.000 & 18.42 & 1.479 & 15.76 & 23.309 \\
\hline 0.062 & 14.29 & 31.832 & 7.34 & 233.647 & 0.031 & 16.56 & 241.470 & 6.55 & 1581.628 & 2.000 & 18.42 & 1.347 & 15.38 & 20.717 \\
\hline 0.062 & 14.31 & 28.441 & 7.42 & 211.032 & 0.031 & 16.58 & 167.820 & 8.22 & 1379.480 & 2.000 & 18.57 & 1.564 & 15.77 & 24.664 \\
\hline 0.062 & 14.31 & 40.563 & 8.56 & 347.219 & 0.031 & 16.78 & 251.500 & 6.15 & 1546.725 & 2.000 & 18.65 & 1.735 & 16.04 & 27.829 \\
\hline 0.062 & 14.41 & 22.417 & 7.77 & 174.180 & 4.000 & 16.91 & 0.720 & 15.83 & 11.398 & 2.000 & 18.80 & 1.574 & 16.16 & 25.436 \\
\hline 0.062 & 14.45 & 29.905 & 7.68 & 229.670 & 4.000 & 16.94 & 0.218 & 15.36 & 3.348 & 2.000 & 19.11 & 2.070 & 16.37 & 33.886 \\
\hline 0.062 & 14.57 & 33.415 & 7.81 & 260.971 & 4.000 & 17.04 & 1.195 & 15.55 & 18.582 & 2.000 & 19.39 & 3.400 & 16.46 & 55.964 \\
\hline 0.062 & 14.59 & 36.743 & 8.22 & 302.027 & 4.000 & 17.07 & 0.250 & 15.71 & 3.927 & 2.000 & 19.46 & 1.238 & 16.21 & 20.068 \\
\hline 0.062 & 14.66 & 53.194 & 8.26 & 439.382 & 4.000 & 17.11 & 0.855 & 15.85 & 13.552 & 2.000 & 19.66 & 1.320 & 16.43 & 21.688 \\
\hline 0.062 & 14.86 & 44.899 & 8.69 & 390.172 & 4.000 & 17.20 & 0.746 & 15.95 & 11.899 & 2.000 & 19.81 & 2.225 & 16.70 & 37.157 \\
\hline
\end{tabular}




\begin{tabular}{|c|c|c|c|c|c|c|c|c|c|c|c|c|c|c|}
\hline $\begin{array}{l}\text { He ight } \\
(z)\end{array}$ & $\begin{array}{l}\text { Wind } \\
\text { e } 10 \mathrm{~m}\end{array}$ & $\begin{array}{l}\text { Mass } \\
\text { Conc }\end{array}$ & $\begin{array}{l}\text { Wind } \\
\text { e } \mathrm{zm}\end{array}$ & $\begin{array}{l}\text { Mess } \\
\text { Flux }\end{array}$ & $\begin{array}{l}\text { Height } \\
\text { (z) }\end{array}$ & $\begin{array}{l}\text { wind } \\
\text { e } 10 \mathrm{~m}\end{array}$ & $\begin{array}{l}\text { Mass } \\
\text { Conc }\end{array}$ & $\begin{array}{l}\text { Wind } \\
e z m\end{array}$ & $\begin{array}{l}\text { Mass } \\
\text { Flux }\end{array}$ & $\begin{array}{l}\text { He ight } \\
(z)\end{array}$ & $e^{\text {Wind }}$ & $\begin{array}{l}\text { Mass } \\
\text { Cone }\end{array}$ & $\begin{array}{l}\text { Wind } \\
a \geq m\end{array}$ & $\begin{array}{l}\text { Mass } \\
\text { Flux }\end{array}$ \\
\hline 1.000 & 16.91 & 2.515 & 13.49 & 33.927 & 0.500 & 18.80 & 6.151 & 13.64 & 83.900 & 0.125 & 18.21 & 27.326 & 11.32 & 309.330 \\
\hline 1.000 & 16.94 & 1.184 & 13.43 & 15.901 & 0.500 & 19.11 & 8.443 & 13.87 & 117.104 & 0.125 & 18.39 & 25.091 & 10.82 & 271.485 \\
\hline 1.000 & 17.04 & 4.468 & 13.53 & 60.452 & 0.500 & 19.39 & 13.294 & 14.00 & 186.116 & 0.125 & 18.42 & 32.057 & 10.63 & 340.766 \\
\hline 1.000 & 17.07 & 1.316 & 13.71 & 18.042 & 0.500 & 19.46 & 5.931 & 13.83 & 82.026 & 0.125 & 18.42 & 34.964 & 10.26 & 358.731 \\
\hline 1.000 & 17.11 & 3.037 & 13.66 & 41.485 & 0.500 & 19.66 & 6.050 & 13.82 & 83.611 & 0.125 & 18.57 & 40.048 & 10.99 & 440.127 \\
\hline 1.000 & 17.20 & 2.665 & 13.66 & 36.404 & 0.500 & 19.81 & 9.921 & 13.92 & 138.100 & 0.125 & 18.55 & 24.204 & 11.35 & 274.715 \\
\hline 1.000 & 17.25 & 1.373 & 13.96 & 19.167 & 0.250 & 16.91 & 11.430 & 11.54 & 131.902 & 0.125 & 18.80 & 29.962 & 11.32 & 339.170 \\
\hline 1.000 & 17.35 & 2.978 & 13.30 & 39.607 & 0.250 & 16.94 & 8.266 & 10.97 & 90.678 & 0.125 & 19.11 & 38.084 & 11.38 & 433.396 \\
\hline 1.000 & 17.48 & 2.798 & 13.43 & 37.577 & 0.250 & 17.04 & 16.482 & 11.45 & 188.719 & 0.125 & 19.39 & 55.945 & 11.54 & 645.605 \\
\hline 1.000 & 17.51 & 1.473 & 14.17 & 20.872 & 0.250 & 17.07 & 5.813 & 11.56 & 67.198 & 0.125 & 19.46 & 33.078 & 10.93 & 361.543 \\
\hline 1.000 & 17.51 & 3.135 & 13.64 & 42.761 & 0.250 & 17.11 & 14.095 & 11.70 & 164.911 & 0.125 & 19.66 & 33.624 & 11.27 & 378.942 \\
\hline 1.000 & 17.72 & 3.708 & 14.10 & 52.283 & 0.250 & 17.20 & 10.714 & 19.62 & 124.497 & 0.125 & 19.81 & 55.037 & 11.20 & 616.414 \\
\hline 1.000 & 17.76 & 3.331 & 13.87 & 46.201 & 0.250 & 17.25 & 7.125 & 11.88 & 84.645 & 0.062 & 16.91 & 47.530 & 9.79 & 465.319 \\
\hline 1.000 & 17.79 & 2.844 & 13.67 & 38.877 & 0.250 & 17.35 & 12.686 & 10.94 & 138.785 & 0.062 & 16.94 & 66.010 & 8.86 & 584.849 \\
\hline 1.000 & 17.92 & 3.706 & 13.34 & 49.438 & 0.250 & 17.48 & 10.207 & 11.30 & 115.339 & 0.062 & 17.04 & 71.910 & 9.41 & 676.673 \\
\hline 1.000 & 17.98 & 1.947 & 13.84 & 26.946 & 0.250 & 17.51 & 7.134 & 12.07 & 86.107 & 0.062 & 17.07 & 29.980 & 9.53 & 285.709 \\
\hline 1.000 & 18.02 & 3.319 & 14.08 & 46.732 & 0.250 & 17.51 & 12.376 & 11.23 & 138.982 & 0.062 & 17.11 & 88.930 & 9.57 & 851.060 \\
\hline 1.000 & 18.20 & 3.264 & 13.78 & 44.978 & 0.250 & 17.72 & 17.221 & 11.44 & 197.008 & 0.062 & 17.20 & 51.690 & 9.67 & 499.842 \\
\hline 1.000 & 18.21 & 3.578 & 14.30 & 51.165 & 0.250 & 17.76 & 16.013 & 11.70 & 187.352 & 0.062 & 17.25 & 41.630 & 9.86 & 410.472 \\
\hline 1.000 & 18.39 & 1.683 & 14.44 & 24.303 & 0.250 & 17.79 & 11.787 & 11.62 & 136.965 & 0.062 & 17.35 & 82.430 & 8.53 & 703.128 \\
\hline 1.000 & 18.42 & 3.017 & 14.21 & 42.872 & 0.250 & 17.92 & 17.171 & 11.82 & 202.961 & 0.062 & 17.48 & 66.480 & 8.28 & 550.454 \\
\hline 1.000 & 18.42 & 2.781 & 13.96 & 38.823 & 0.250 & 17.98 & 9.126 & 11.49 & 104.858 & 0.062 & 17.51 & 75.970 & 9.99 & 758.940 \\
\hline 1.000 & 18.57 & 2.822 & 14.59 & 41.173 & 0.250 & 18.02 & 15.257 & 11.56 & 176.371 & 0.062 & 17.51 & 72.580 & 8.88 & 644.510 \\
\hline 1.000 & 18.65 & 3.134 & 14.97 & 46.916 & 0.250 & 18.20 & 13.343 & 11.63 & 155.179 & 0.062 & 17.72 & 76.360 & 8.81 & 672.731 \\
\hline 1.000 & 18.80 & 3.020 & 14.88 & 44.938 & 0.250 & 18.21 & 15.380 & 12.30 & 189.174 & 0.062 & 17.76 & 83.310 & 9.44 & 786.446 \\
\hline 1.000 & 19.11 & 3.730 & 15.05 & 56.136 & 0.250 & 10.39 & 10.313 & 12.01 & 123.859 & 0.062 & 17.79 & 77.000 & 9.04 & 696.080 \\
\hline 1.000 & 19.39 & 6.471 & 15.27 & 98.812 & 0.250 & 18.42 & 15.289 & 11.92 & 182.245 & 0.062 & 17.92 & 74.060 & 7.80 & 577.668 \\
\hline 1.000 & 19.46 & 2.748 & 14.90 & 40.945 & 0.250 & 18.42 & 17.401 & 11.80 & 205.332 & 0.062 & 17.98 & 76.410 & 8.72 & 666.295 \\
\hline 1.000 & 19.66 & 3.313 & 15.35 & 50.855 & 0.250 & 18.57 & 12.588 & 12.42 & 156.343 & 0.062 & 18.02 & 62.460 & 8.60 & 537.156 \\
\hline 1.000 & 19.81 & 4.740 & 15.10 & 71.574 & 0.250 & 18.65 & 14.611 & 12.44 & 181.761 & 0.062 & 18.20 & 97.060 & 8.78 & 852.187 \\
\hline 0.500 & 16.91 & 5.601 & 12.63 & 70.741 & 0.250 & 18.80 & 13.956 & 12.51 & 174.590 & 0.062 & 18.21 & 56.900 & 10.41 & 592.329 \\
\hline 0.500 & 16.94 & 2.858 & 12.22 & 34.925 & 0.250 & 19.11 & 19.678 & 12.69 & 249.714 & 0.062 & 18.39 & 76.250 & 9.84 & 750.300 \\
\hline 0.500 & 17.04 & 8.781 & 12.56 & 110.289 & 0.250 & 19.39 & 26.268 & 12.81 & 335.493 & 0.062 & 18.42 & 86.530 & 9.40 & 813.382 \\
\hline 0.500 & 17.07 & 2.536 & 12.64 & 32.055 & 0.250 & 19.46 & 17.433 & 12.54 & 218.610 & 0.062 & 18.42 & 88.430 & 8.97 & 793.217 \\
\hline 0.500 & 17.11 & 6.030 & 12.62 & 76.099 & 0.250 & 19.66 & 14.168 & 12.66 & 179.367 & 0.062 & 18.57 & 84.480 & 9.51 & 803.405 \\
\hline 0.500 & 17.20 & 4.930 & 12.67 & 62.463 & 0.250 & 19.81 & 22.125 & 12.55 & 277.669 & 0.062 & 18.65 & 58.980 & 10.19 & 601.006 \\
\hline 0.500 & 17.25 & 3.179 & 12.90 & 41.009 & 0.125 & 16.91 & 20.558 & 10.65 & 218.943 & 0.062 & 18.80 & 61.510 & 10.14 & 623.711 \\
\hline 0.500 & 17.35 & 5.827 & 13.30 & 77.499 & 0.125 & 16.94 & 18.864 & 9.97 & 188.074 & 0.062 & 19.11 & 82.330 & 10.07 & 829.063 \\
\hline 0.500 & 17.48 & 4.962 & 12.36 & 61.330 & 0.125 & 17.04 & 37.033 & 10.45 & 386.995 & 0.062 & 19.39 & 87.750 & 10.30 & 903.825 \\
\hline 0.500 & 17.51 & 3.050 & 13.05 & 39.802 & 0.125 & 17.07 & 13.929 & 10.56 & 147.090 & 0.062 & 19.46 & 104.850 & 9.34 & 979.299 \\
\hline 0.500 & 17.51 & 6.142 & 12.43 & 76.345 & 0.125 & 17.11 & 24.151 & 10.60 & 256.001 & 0.062 & 19.66 & 89.630 & 9.94 & 890.922 \\
\hline 0.500 & 17.72 & 7.995 & 12.98 & 103.775 & 0.125 & 17.20 & 22.179 & 10.53 & 233.545 & 0.062 & 19.81 & 113.930 & 9.89 & 1126.767 \\
\hline 0.500 & 17.76 & 7.078 & 13.00 & 92.014 & 0.125 & 17.25 & 14.706 & 10.86 & 159.707 & 0.031 & 16.91 & 101.720 & 8.67 & 881.912 \\
\hline 0.500 & 17.79 & 5.867 & 12.46 & 73.103 & 0.125 & 17.35 & 29.119 & 10.90 & 317.397 & 0.031 & 16.94 & 227.150 & 7.77 & 1764.955 \\
\hline 0.500 & 17.92 & 7.611 & 13.34 & 101.531 & 0.125 & 17.48 & 24.256 & 9.79 & 237.466 & 0.031 & 17.04 & 182.740 & 8.36 & 1527.706 \\
\hline 0.500 & 17.98 & 3.743 & 12.66 & 47.386 & 0.125 & 17.51 & 30.899 & 11.01 & 340.198 & 0.031 & 17.07 & 107.200 & 8.51 & 912.272 \\
\hline 0.500 & 18.02 & 6.094 & 12.94 & 78.856 & 0.125 & 17.51 & 29.703 & 10.08 & 299.406 & 0.031 & 17.11 & 184.450 & 8.54 & 1575.203 \\
\hline 0.500 & 18.20 & 7.128 & 12.40 & 88.387 & 0.125 & 17.72 & 30.149 & 10.80 & 325.609 & 0.031 & 17.20 & 130.010 & 8.42 & 1094.684 \\
\hline 0.500 & 18.21 & 7.153 & 13.50 & 96.565 & 0.125 & 17.76 & 32.898 & 10.58 & 348.061 & 0.031 & 17.25 & 102.910 & 8.86 & 911.783 \\
\hline 0.500 & 18.39 & 3.720 & 13.10 & 48.732 & 0.125 & 17.79 & 36.731 & 10.24 & 376.125 & 0.031 & 17.35 & 170.890 & 7.01 & 1197.939 \\
\hline 0.500 & 18.42 & 5.914 & 13.03 & 77.059 & 0.125 & 17.92 & 40.607 & 10.64 & 432.058 & 0.031 & 17.48 & 181.780 & 7.35 & 1336.083 \\
\hline 0.500 & 18.42 & 6.733 & 12.79 & 86.115 & 0.125 & 17.98 & 26.737 & 10.11 & 270.311 & 0.031 & 17.51 & 219.920 & 8.96 & 1970.483 \\
\hline 0.500 & 18.57 & 6.093 & 13.65 & 83.169 & 0.125 & 18.02 & 33.728 & 10.03 & 338.292 & 0.031 & 17.51 & 213.310 & 7.72 & 1646.753 \\
\hline 0.500 & 18.65 & 6.630 & 13.61 & 90.234 & 0.125 & 18.20 & 40.139 & 10.07 & 404.200 & 0.031 & 17.72 & 257.870 & 8.61 & 2220.261 \\
\hline
\end{tabular}




\begin{tabular}{|c|c|c|c|c|c|c|c|c|c|c|c|c|c|c|}
\hline $\begin{array}{l}\text { He i ght } \\
(z)\end{array}$ & $\begin{array}{l}\text { Wind } \\
e 10 \mathrm{~m}\end{array}$ & $\begin{array}{l}\text { Mass } \\
\text { Conc }\end{array}$ & $\begin{array}{l}\text { Wind } \\
e \approx n\end{array}$ & $\begin{array}{l}\text { Mass } \\
\text { Flux }\end{array}$ & $\begin{array}{l}\text { Heignt } \\
\text { (z) }\end{array}$ & $e^{\text {Wind }} 10 \mathrm{~m}$ & $\begin{array}{l}\text { Mass } \\
\text { Conc }\end{array}$ & $\begin{array}{l}\text { Wind } \\
e z m\end{array}$ & $\begin{array}{l}\text { Mass } \\
\text { Flux }\end{array}$ & $\begin{array}{l}\text { Height } \\
(z)\end{array}$ & $\begin{array}{l}\text { Wind } \\
\text { e } 10 \mathrm{~m}\end{array}$ & $\begin{array}{l}\text { Mass } \\
\text { Conc }\end{array}$ & $\begin{array}{l}\text { Wind } \\
e z \mathrm{~m}\end{array}$ & $\begin{array}{l}\text { Mass } \\
\text { Flux }\end{array}$ \\
\hline 0.031 & 17.76 & 216.180 & 8.31 & 1796.456 & 0.500 & 20.17 & 6.873 & 14.36 & 98.696 & 0.031 & 22.43 & 371.510 & 9.95 & 3696.524 \\
\hline 0.031 & 17.79 & 190.230 & 7.85 & 1493.305 & 0.500 & 20.95 & 14.799 & 15.07 & 223.021 & 0.031 & 22.62 & 276.900 & 11.10 & 3073.590 \\
\hline 0.031 & 17.92 & 277.290 & 6.42 & 1780.202 & 0.500 & 20.96 & 11.577 & 15.16 & 175.507 & 0.031 & 22.88 & $5,43.700$ & 9.64 & 5241.267 \\
\hline 0.031 & 17.98 & 198.470 & 7.63 & 1514.326 & 0.500 & 22.08 & 15.298 & 15.69 & 240.026 & 0.031 & 23.51 & 505.410 & 10.49 & 5301.750 \\
\hline 0.031 & 18.02 & 233.880 & 7.62 & 1782.166 & 0.500 & 22.13 & 19.601 & 15.85 & 310.676 & 0.031 & 23.94 & 544.590 & 10.36 & 5641.952 \\
\hline 0.031 & 18.20 & 298.660 & 7.50 & 2239.950 & 0.500 & 22.20 & 16.199 & 15.06 & 243.957 & 0.031 & 24.02 & 348.970 & 9.97 & 3479.230 \\
\hline 0.031 & 18.21 & 148.660 & 9.15 & 1360.239 & 0.500 & 22.43 & 11.627 & 16.25 & 188.939 & 4.000 & 20.13 & 2.461 & 18.55 & 45.652 \\
\hline 0.031 & 18.39 & 235.310 & 8.43 & 1983.663 & 0.500 & 22.62 & 11.943 & 16.48 & 196.821 & 4.000 & 14.74 & 0.428 & 13.76 & 5.889 \\
\hline 0.031 & 18.42. & 264.940 & 8.16 & 2161.910 & 0.500 & 22.88 & 13.579 & 15.79 & 214.412 & 4.000 & 22.94 & 2.030 & 21.49 & 43.625 \\
\hline 0.031 & 18.42 & 262.050 & 7.68 & 2012.544 & 0.500 & 23.51 & 21.115 & 16.59 & 350.298 & 4.000 & 21.69 & 6.670 & 20.00 & 133.400 \\
\hline 0.031 & 18.57 & 300.630 & 8.59 & 2582.412 & 0.500 & 23.94 & 19.057 & 16.75 & 319.205 & 4.000 & 26.89 & 11.250 & 25.00 & 281.250 \\
\hline 0.031 & 18.65 & 139.100 & 9.04 & 1257.464 & 0.500 & 24.02 & 21.644 & 16.60 & 359.290 & 4.000 & 29.23 & 10.410 & 27.34 & 284.609 \\
\hline 0.031 & 18.80 & 173.860 & 8.96 & 1557.785 & 0.250 & 20.17 & 15.010 & 13.33 & 200.083 & 4.000 & 20.91 & 1.890 & 19.22 & 36.326 \\
\hline 0.031 & 19.11 & 233.600 & 8.94 & 2088.384 & 0.250 & 20.95 & 29.170 & 14.04 & 409.547 & 4.000 & 24.28 & 4.020 & 22.31 & 89.686 \\
\hline 0.031 & 19.39 & 227.530 & 9.06 & 2061.421 & 0.250 & 20.96 & 23.880 & 14.36 & 342.917 & 4.000 & 24.60 & 3.520 & 22.91 & 80.643 \\
\hline 0.031 & 19.46 & 338.060 & 8.23 & 2782.233 & 0.250 & 22.08 & 32.060 & 14.27 & 457.496 & 4.000 & 23.27 & 2.220 & 21.97 & 48.773 \\
\hline 0.031 & 19.66 & 475.160 & 8.62 & 4095.879 & 0.250 & 22.13 & 42.090 & 14.18 & 596.836 & 4.000 & 26.27 & 6.490 & 24.92 & 161.731 \\
\hline 0.031 & 19.81 & 355.920 & 8.47 & 3014.642 & 0.250 & 22.20 & 36.000 & 13.43 & 483.480 & 4.000 & 27.75 & 4.460 & 25.78 & 114.979 \\
\hline 4.000 & 20.17 & 0.973 & 18.36 & 17.864 & 0.250 & 22.43 & 28.860 & 14.21 & 410.101 & 4.000 & 21.95 & 1.270 & 19.97 & 25.362 \\
\hline 4.000 & 20.95 & 1.870 & 19.12 & 35.754 & 0.250 & 22.62 & 24.400 & 15.50 & 378.200 & 4.000 & 16.68 & 0.293 & 15.49 & 4.539 \\
\hline 4.000 & 20.96 & 1.574 & 19.30 & 30.378 & 0.250 & 22.88 & 30.030 & 14.55 & 436.936 & 4.000 & 24.04 & 2.361 & 22.43 & 52.957 \\
\hline 4.000 & 22.08 & 1.950 & 20.06 & 39.117 & 0.250 & 23.51 & 41.350 & 15.37 & 635.549 & 4.000 & 23.07 & 0.771 & 21.73 & 16.754 \\
\hline 4.000 & 22.13 & 2.335 & 20.20 & 47.167 & 0.250 & 23.94 & 38.100 & 15.45 & 588.645 & 4.000 & 25.44 & 2.990 & 23.88 & 71.401 \\
\hline 4.000 & 22.20 & 2.242 & 19.85 & 44.504 & 0.250 & 24.02 & 44.340 & 15.09 & 669.090 & 4.000 & 18.22 & 0.262 & 17.28 & 4.527 \\
\hline 4.000 & 22.43 & 1.432 & 20.29 & 29.055 & 0.125 & 20.17 & 38.080 & 11.94 & 454.675 & 2.000 & 20.13 & 4.142 & 17.31 & 71.698 \\
\hline 4.000 & 22.62 & 1.944 & 20.87 & 40.571 & 0.125 & 20.95 & 58.630 & 12.62 & 739.911 & 2.000 & 14.74 & 6.599 & 11.82 & 78.000 \\
\hline 4.000 & 22.88 & 1.783 & 20.78 & 37.051 & 0.125 & 20.96 & 44.390 & 12.86 & 570.855 & 2.000 & 22.94 & 4.540 & 20.48 & 92.979 \\
\hline 4.000 & 23.51 & 2.828 & 21.57 & 61.000 & 0.125 & 22.08 & 62.550 & 12.87 & 805.019 & 2.000 & 21.69 & 10.160 & 18.90 & 192.024 \\
\hline 4.000 & 23.94 & 2.861 & 21.87 & 62.570 & 0.125 & 22.13 & 69.070 & 12.89 & 890.312 & 2.000 & 26.89 & 17.350 & 23.69 & 411.021 \\
\hline 4.000 & 24.02 & 2.782 & 21.82 & 60.703 & 0.125 & 22.20 & 63.590 & 11.83 & 752.270 & 2.000 & 29.23 & 13.310 & 26.00 & 346.060 \\
\hline 2.000 & 20.17 & 1.572 & 17.32 & 27.227 & 0.125 & 22.43 & 80.050 & 12.37 & 990.219 & 2.000 & 20.91 & 3.520 & 18.18 & 63.994 \\
\hline 2.000 & 20.95 & 4.017 & 18.09 & 22.668 & 0.125 & 22.62 & 70.790 & 13.87 & 981.857 & 2.000 & 24.28 & 6.680 & 20.96 & 140.013 \\
\hline 2.000 & 20.96 & 3.279 & 18.08 & 59.284 & 0.125 & 22.88 & 89.070 & 12.78 & 1138.315 & 2.000 & 24.60 & 7.420 & 21.70 & 161.014 \\
\hline 2.000 & 22.08 & 3.244 & 18.68 & 60.598 & 0.125 & 23.51 & 72.700 & 13.62 & 990.174 & 2.000 & 23.27 & 3.740 & 21.12 & 78.989 \\
\hline 2.000 & 22.13 & 4.947 & 18.77 & 92.855 & 0.125 & 23.94 & 76.170 & 13.62 & 1037.435 & 2.000 & 26.27 & 9.230 & 24.27 & 224.012 \\
\hline 2.000 & 22.20 & 4.029 & 18.62 & 75.020 & 0.125 & 24.02 & 80.300 & 13.36 & 1072.808 & 2.000 & 27.75 & 7.540 & $24.40^{\circ}$ & 183.976 \\
\hline 2.000 & 22.43 & 2.503 & 18.73 & 46.881 & 0.062 & 20.17 & 89.110 & 9.31 & 829.614 & 2.000 & 21.95 & 3.830 & 18.62 & 71.315 \\
\hline 2.000 & 22.62 & 3.210 & 19.59 & 62.884 & 0.062 & 20.95 & 145.560 & 11.30 & 1644.828 & 2.000 & 16.68 & 0.524 & 14.81 & 7.760 \\
\hline 2.000 & 22.88 & 3.511 & 19.36 & 67.973 & 0.062 & 20.96 & 99.480 & 11.58 & 1151.978 & 2.000 & 24.04 & 4.730 & 21.56 & 101.979 \\
\hline 2.000 & 23.51 & 6.185 & 19.83 & 122.649 & 0.062 & 22.08 & 141.400 & 11.41 & 1613.374 & 2.000 & 23.07 & 2.267 & 20.16 & 45.703 \\
\hline 2.000 & 23.94 & 6.686 & 20.23 & 135.258 & 0.062 & 22.13 & 126.430 & 11.51 & 1455.209 & 2.000 & 25.44 & 4.180 & 22.92 & 95.806 \\
\hline 2.000 & 24.02 & 5.279 & 20.00 & 105.580 & 0.062 & 22.20 & 160.430 & 10.19 & 1634.781 & 2.000 & 18.22 & 0.464 & 16.72 & 7.758 \\
\hline 1.000 & 20.17 & 3.261 & 15.84 & 51.654 & 0.062 & 22.43 & 168.410 & 9.22 & 1552.740 & 1.000 & 20.13 & 6.609 & 16.06 & 106.141 \\
\hline 1.000 & 20.95 & 7.454 & 16.53 & 123.215 & 0.062 & 22.62 & 131.300 & 12.48 & 1638.624 & 1.000 & 14.74 & 1.261 & 12.31 & 15.523 \\
\hline 1.000 & 20.96 & 6.101 & 16.62 & 101.399 & 0.062 & 22.88 & 189.220 & 9.57 & 1810.835 & 1.000 & 22.94 & 5.010 & 19.55 & 97.945 \\
\hline 1.000 & 22.08 & 7.164 & 17.48 & 125.227 & 0.062 & 23.51 & 136.430 & 12.06 & 1645.345 & 1.000 & 21.69 & 14.710 & 17.88 & 263.015 \\
\hline 1.000 & 22.13 & 9.947 & 17.27 & 171.785 & 0.062 & 23.94 & 198.890 & 11.99 & 2384.691 & 1.000 & 26.89 & 23.170 & 22.44 & 519.935 \\
\hline 1.000 & 22.20 & 7.507 & 16.79 & 126.043 & 0.062 & 24.02 & 151.530 & 11.67 & 1768.355 & 1.000 & 29.23 & 22.570 & 24.69 & 557.253 \\
\hline 1.000 & 22.43 & 5.715 & 17.26 & 98.641 & 0.031 & 20.17 & 310.310 & 9.33 & 2895.192 & 1.000 & 20.91 & 6.450 & 17.26 & 111.327 \\
\hline 1.000 & 22.62 & 7.009 & 17.68 & 123.919 & 0.031 & 20.95 & 365.630 & 9.98 & 3648.987 & 1.000 & 24.28 & 11.610 & 19.74 & 229.181 \\
\hline 1.000 & 22.88 & 6.603 & 17.30 & 114.232 & 0.031 & 20.96 & 365.450 & 10.30 & 3764.135 & 1.000 & 24.60 & 11.530 & 20.56 & 237.057 \\
\hline 1.000 & 23.51 & 11.038 & 18.21 & 201.002 & 0.031 & 22.08 & 406.340 & 9.95 & 4043.083 & 1.000 & 23.27 & 6.180 & 20.31 & 125.516 \\
\hline 1.000 & 23.94 & 10.367 & 18.28 & 189.509 & 0.031 & 22.13 & 299.290 & 9.96 & 2980.928 & 1.000 & 26.27 & 12.300 & 23.83 & 293.109 \\
\hline 1.000 & 24.02 & 10.881 & 18.57 & 202.060 & 0.031 & 22.20 & 382.750 & 8.55 & 3272.512 & 1.000 & 27.75 & 13.320 & 23.10 & 307.692 \\
\hline
\end{tabular}




\begin{tabular}{|c|c|c|c|c|c|c|c|c|c|c|c|c|c|c|c|}
\hline & $\begin{array}{l}\text { He ight } \\
(z)\end{array}$ & $e^{\text {Wind }}$ & $\begin{array}{l}\text { Mass } \\
\text { Conc }\end{array}$ & $\begin{array}{l}\text { Wind } \\
\text { e } \mathrm{zm}\end{array}$ & $\begin{array}{l}\text { Mass } \\
\text { Flux }\end{array}$ & $\begin{array}{l}\text { Height } \\
(z)\end{array}$ & $\begin{array}{l}\text { Wind } \\
\text { e } 10 \mathrm{~m}\end{array}$ & $\begin{array}{l}\text { Mass } \\
\text { Conic }\end{array}$ & $\begin{array}{l}\text { Wind } \\
\text { e } \mathrm{zm}\end{array}$ & $\begin{array}{l}\text { Mass } \\
\text { Flux }\end{array}$ & $\begin{array}{l}\text { Height } \\
(z)\end{array}$ & $\begin{array}{l}\text { Wind } \\
e^{10 \mathrm{~m}}\end{array}$ & $\begin{array}{l}\text { Mass } \\
\text { Conc }\end{array}$ & $\begin{array}{l}\text { Wind } \\
\text { e } \mathrm{z} \mathrm{m}\end{array}$ & $\begin{array}{l}\text { Mass } \\
\text { Flux }\end{array}$ \\
\hline & 1.000 & 21.95 & 5.980 & 17.40 & 104.052 & 0.120 & 21.95 & 82.750 & 12.93 & 1069.958 & 1.000 & 25.00 & 15.200 & 18.00 & 273.600 \\
\hline & 1.000 & 16.68 & 1.300 & 14.22 & 18.486 & 0.120 & 16.68 & 50.870 & 11.56 & 588.057 & 1.000 & 33.00 & 15.900 & 23.80 & 378.420 \\
\hline & 1.000 & 24.04 & 7.091 & 20.78 & 147.351 & 0.120 & 24.04 & 104.700 & 17.13 & 1793.511 & 1.000 & 36.00 & 13.500 & .25 .90 & 349.650 \\
\hline & 1.000 & 23.07 & 2.333 & 20.58 & 48.013 & 0.120 & 23.07 & 48.320 & 17.59 & 849.949 & 0.500 & 23.00 & 3.580 & 15.00 & 53.700 \\
\hline & 1.000 & 25.44 & 5.850 & 22.12 & 129.402 & 0.120 & 25.44 & 54.700 & 18.61 & 1017.967 & 0.500 & 24.00 & 7.300 & 15.70 & 114.610 \\
\hline & 1.000 & 18.22 & 0.567 & 16.31 & 9.248 & 0.120 & 18.22 & 22.700 & 14.23 & 323.021 & 0.500 & 25.00 & 19.750 & 16.30 & 321.925 \\
\hline & 0.500 & 20.13 & 11.660 & 14.89 & 173.617 & 0.060 & 20.13 & 99.380 & 11.24 & 1117.031 & 0.500 & 33.00 & 18.700 & 21.60 & 403.920 \\
\hline & 0.500 & 14.74 & 1.974 & 11.55 & 22.800 & 0.060 & 14.74 & 33.400 & 9.34 & 311.956 & 0.500 & 36.00 & 17.400 & 23.50 & 408.900 \\
\hline & 0.500 & 22.94 & 7.780 & 18.38 & 142.996 & 0.060 & 22.94 & 96.980 & 15.22 & 1476.035 & 0.040 & 23.00 & 17.050 & 10.50 & 179.025 \\
\hline & 0.500 & 21.69 & 23.500 & 16.47 & 387.045 & 0.060 & 21.69 & 328.400 & 12.85 & 4219.940 & 0.040 & 24.00 & 53.100 & 10.90 & 578.790 \\
\hline & 0.500 & 26.89 & 32.220 & 23.53 & 758.136 & 0.060 & 26.89 & 234.500 & 16.80 & 3939.600 & 0.040 & 25.00 & 136.600 & 11.40 & 1557.240 \\
\hline & 0.500 & 29.23 & 33.980 & 23.19 & 787.996 & 0.060 & 29.23 & 200.800 & 19.02 & 3819.216 & 0.040 & 33.00 & 126.000 & 15.20 & 1915.200 \\
\hline & 0.500 & 20.91 & 10.120 & 15.81 & 159.997 & 0.060 & 20.91 & 207.800 & 12.27 & 2549.706 & 0.040 & 36.00 & 88.800 & 16.40 & 1456.320 \\
\hline & 0.500 & 24.28 & 22.120 & 18.31 & 405.017 & 0.060 & 24.28 & 389.600 & 13.86 & 5399.855 & & & & & \\
\hline & 0.500 & 24.60 & 19.680 & 19.21 & 378.053 & 0.060 & 24.60 & 189.400 & 15.47 & 2930.018 & & & & & \\
\hline & 0.500 & 23.27 & 10.910 & 19.25 & 210.017 & 0.060 & 23.27 & 58.990 & 16.46 & 970.975 & & & 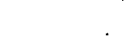 & & \\
\hline & 0.500 & 26.27 & 20.130 & 22.55 & 453.931 & 0.060 & 26.27 & 134.700 & $19.97^{\circ}$ & 2689.959 & & & & & \\
\hline & 0.500 & 27.75 & 23.590 & 21.53 & 507.893 & 0.060 & 27.75 & 272.900 & 17.22 & 4699.338 & & & & & \\
\hline & 0.500 & 21.95 & 13.120 & 15.78 & 207.034 & 0.060 & 21.95 & 204.400 & 11.50 & 2350.600 & & & & & \\
\hline & 0.500 & 16.68 & 3.360 & 13.18 & 44.285 & 0.060 & 16.68 & 150.700 & 10.75 & 1620.025 & & & & & \\
\hline & 0.500 & 24.04 & 14.110 & 18.21 & 256.943 & 0.060 & 24.04 & 320.700 & 16.03 & 5140.820 & & & & & \\
\hline & 0.500 & 23.07 & 4.017 & 19.32 & 77.608 & 0.060 & 23.07 & 120.500 & 16.72 & 2014.760 & & & & & \\
\hline & 0.500 & 25.44 & 10.700 & 20.09 & 214.963 & 0.060 & 25.44 & 94.900 & 17.63 & 1673.087 & & & & & \\
\hline & 0.500 & 18.22 & 0.975 & 15.49 & 15.103 & 0.060 & 18.22 & $50: 300$ & 13.56 & 682.068 & & & & & \\
\hline & 0.250 & 20.13 & 24.470 & 13.69 & 334.994 & 0.030 & 20.13 & 183.900 & 10.02 & 1842.678 & & & & & \\
\hline$\sigma$ & 0.250 & 14.74 & 6.041 & 10.81 & 65.303 & 0.030 & 14.74 & 60.230 & 8.60 & 517.978 & & & & & \\
\hline & 0.250 & 22.94 & 20.890 & 17.27 & 360.770 & 0.030 & 22.94 & 178.000 & 14.16 & 2520.479 & & & & & \\
\hline & 0.250 & 21.69 & 50.720 & -15.17 & 769.422 & 0.030 & 21.69 & 405.500 & 11.64 & 4720.020 & & & & & \\
\hline & 0.250 & 26.89 & 66.650 & 19.49 & 1299.008 & 0.030 & 26.89 & 437.700 & 15.42 & 6749.334 & & & & & \\
\hline & 0.250 & 29.23 & 70.240 & 21.75 & 1527.720 & 0.030 & 29.23 & 536.900 & 17.60 & 9449.439 & & & & & \\
\hline & 0.250 & 20.91 & 28.020 & 14.50 & 406.290 & 0.030 & 20.91 & 391.300 & 11.09 & 4339.517 & & & & & \\
\hline & 0.250 & 24.28 & 50.180 & 16.61 & 833.490 & 0.030 & 24.28 & 822.900 & 12.42 & 10220.418 & & & & & \\
\hline & 0.250 & 24.60 & 45.490 & 17.92 & 815.181 & 0.030 & 24.60 & 254.900 & 14.22 & 3624.678 & & & & & \\
\hline & 0.250 & 23.27 & 22.710 & 18.25 & 414.457 & 0.030 & 23.27 & 103.500 & $15: 54$ & 1608.390 & & & & & \\
\hline & 0.250 & 26.27 & 37.900 & 21.48 & 814.092 & 0.030 & 26.27 & 145.500 & 19.11 & 2780.505 & & & & & \\
\hline & 0.250 & 27.75 & 47.290 & 20.03 & 947.219 & 0.030 & 27.75 & 465.800 & 15.78 & 7350.323 & & & & & \\
\hline & 0.250 & 21.95 & 29.480 & 14.26 & 420.385 & 0.030 & 21.95 & 353.500 & 10.07 & 3559.745 & & & & & \\
\hline & 0.250 & 16.68 & 7.750 & 12.25 & 94.937 & 0.030 & 16.68 & 251.800 & 9.94 & 2502.892 & & & & & \\
\hline & 0.250 & 24.04 & 25.450 & 18.04 & 459.118 & 0.030 & 24.04 & 673.800 & 14.93 & 10059.834 & & & & & \\
\hline & 0.250 & 23.07 & 15.010 & 18.26 & 274.083 & 0.030 & 23.07 & 185.800 & 15.85 & 2944.930 & & & " & & \\
\hline & 0.250 & 25.44 & 20.000 & 19.56 & 391.200 & 0.030 & 25.44 & 137.100 & 16.45 & 2255.295 & & & & & \\
\hline & 0.250 & 18.22 & 5.440 & 14.74 & 80.186 & 0.030 & 18.22 & 91.700 & 12.92 & 1184.764 & & & & & \\
\hline & 0.120 & 20.13 & 43.420 & 12.46 & 541.013 & 4.000 & 23.00 & 1.500 & 20.20 & 30.300 & & & & & \\
\hline & 0.120 & 14.74 & 10.220 & 10.08 & 103.018 & 4.000 & 24.00 & 3.430 & 21.00 & 72.030 & & & & & - \\
\hline & 0.120 & 22.94 & 58.270 & 16.27 & 948.053 & 4.000 & 25.00 & 8.610 & 21.90 & 188.559 & & & . & & \\
\hline & 0.120 & 21.69 & 125.200 & 14.06 & 1760.312 & 4.000 & 33.00 & 11.500 & 28.90 & 332.350 & & & & & \\
\hline & 0.120 & 26.89 & 113.900 & 18.17 & 2069.562 & 4.000 & 36.00 & 8.220 & 31.60 & 259.752 & & & & & \\
\hline & 0.120 & 29.23 & 93.580 & 20.41 & 1909.968 & 2.000 & 23.00 & 1.860 & 18.30 & 34.038 & & & & & \\
\hline & 0.120 & 20.91 & 69.520 & 13.45 & 935.044 & 2.000 & 24.00 & 4.160 & 19.10 & 79.456 & & & & & \\
\hline & 0.120 & 24.28 & 138.800 & 15.27 & 2119.476 & 2.000 & 25.00 & 12.550 & 19.90 & 249.745 & & & & & \\
\hline & 0.120 & 24.60 & 125.000 & 16.72 & 2090.000 & 2.000 & 33.00 & 13.550 & 26.30 & 356.365 & & & & & \\
\hline & 0.120 & 23.27 & 33.980 & 17.39 & 590.912 & 2.000 & 36.00 & 11.120 & 28.60 & 318.032 & & & & & \\
\hline & 0.120 & 26.27 & 56.550 & 20.83 & 1177.937 & 1.000 & 23.00 & 2.860 & 16.60 & 47.476 & & & & & \\
\hline & 0.120 & 27.75 & 115.000 & 18.65 & 2144.750 & 1.000 & 24.00 & 5.930 & 17.30 & 102.589 & & & & & \\
\hline
\end{tabular}

